\title{
A Dynamic Sensor Concept Combining Electrochemical pH Manipulation and Optical Sensing of Buffer Capacity
}

Fabian Steininger ${ }^{\prime \prime}$, Silvia E. Zieger" and Klaus Koren ${ }^{\| *}$

I: Aarhus University Centre for Water Technology (WATEC), Department of Biology, Ny Munkegade 114, 8000 Aarhus C, Denmark

\$: Graz University of Technology, Institute of Analytical Chemistry and Food Chemistry, Stremayrgasse 9/II, 8010 Graz, Austria

Corresponding Author*: klaus.koren@bio.au.dk

Table of Content:

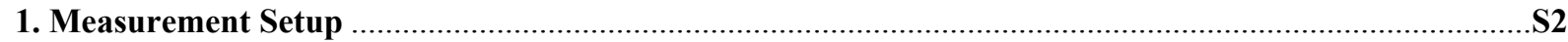

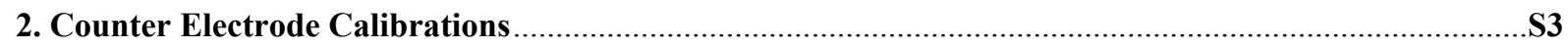

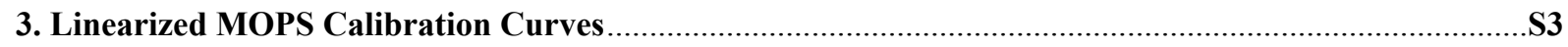

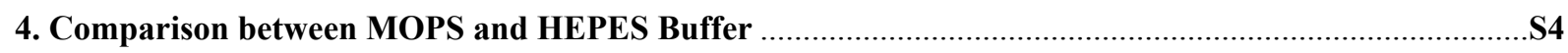

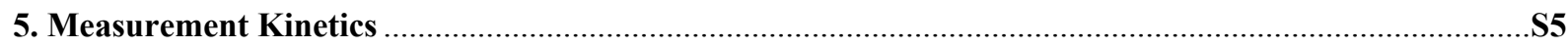

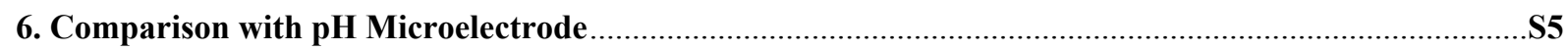

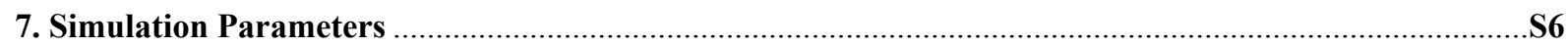




\section{Measurement Setup}

Top View:

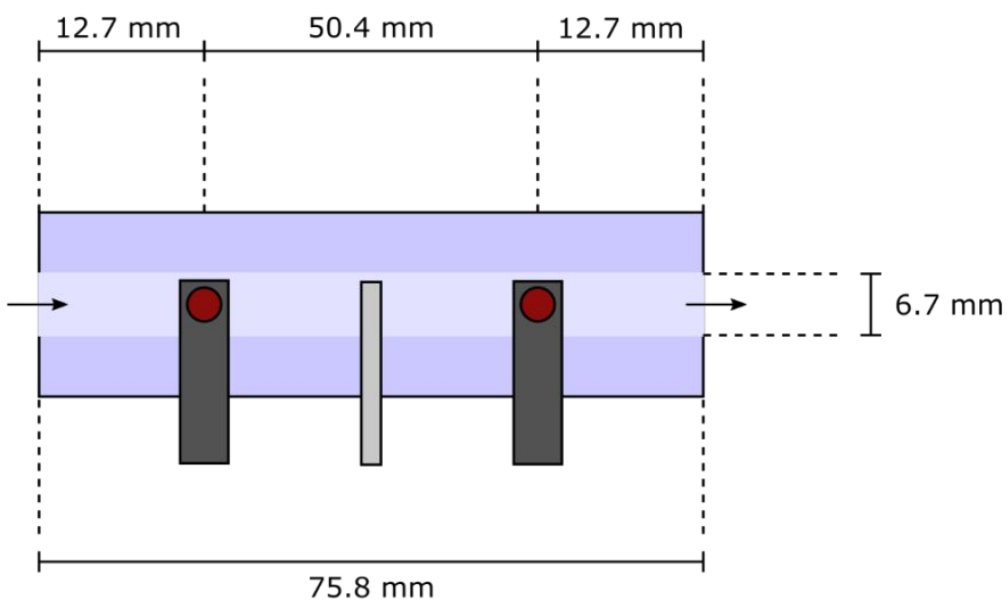

Side View:

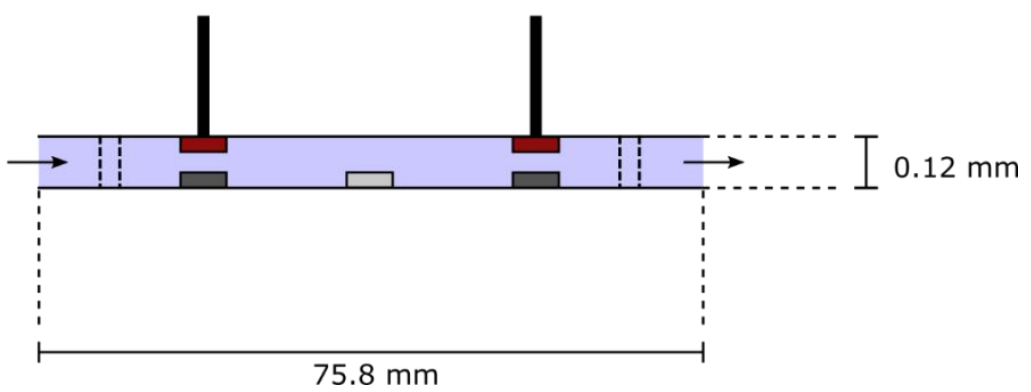

Figure S1. Dimension of measurement cell - top and side view of flow cell.

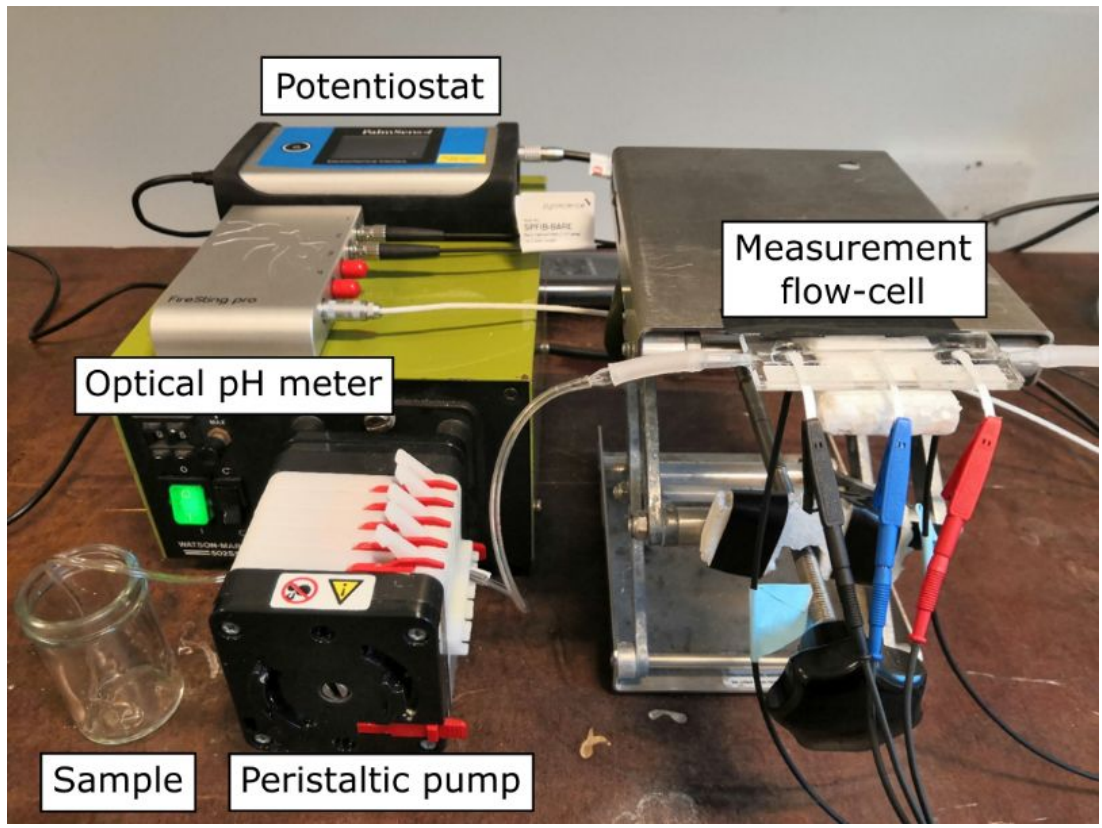

Figure S2. Final measurement setup 


\section{Counter Electrode Calibrations}

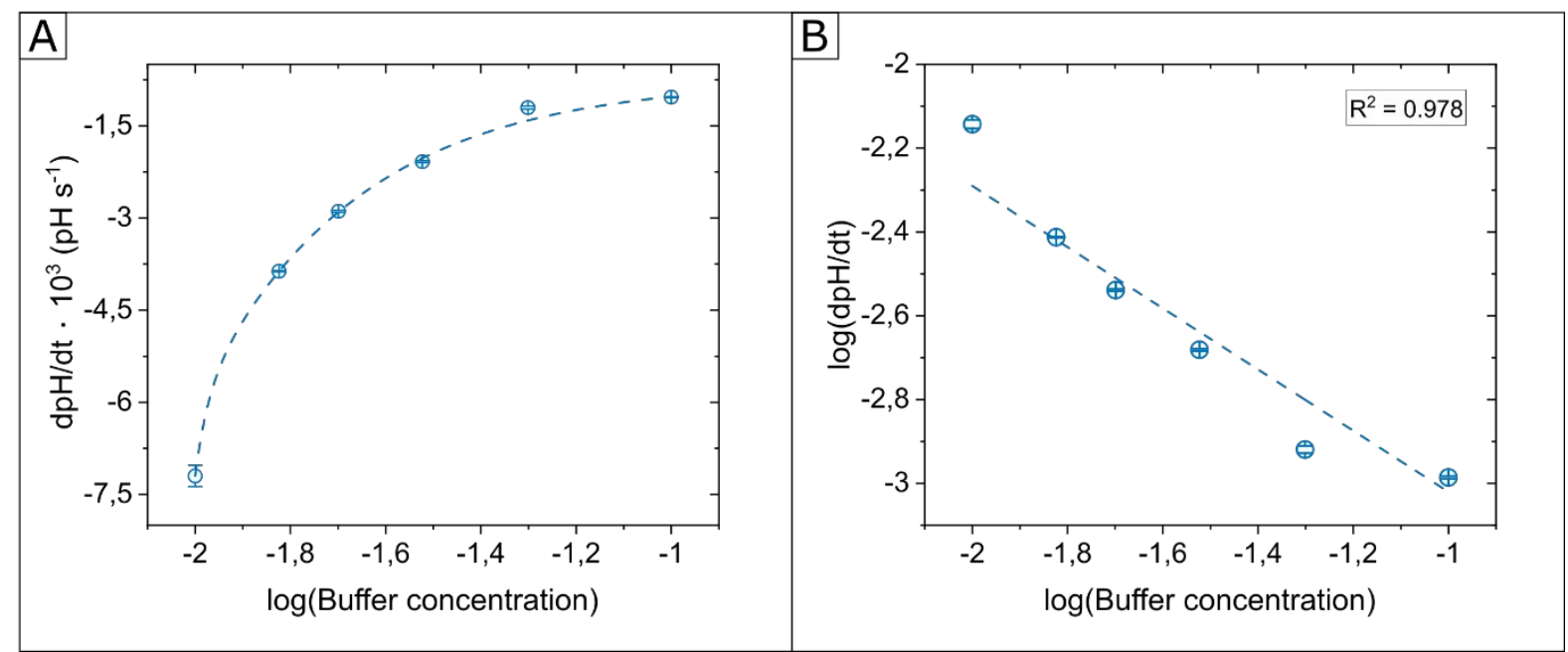

Figures S3. (A) Dynamic calibration of MOPS buffer (counter electrode). (B) Linearized calibration by double logarithmic representation of rate of $\mathrm{pH}$ change and buffer concentration. For the linearization, absolute values for $\mathrm{dpH} / \mathrm{dt}$ were used to form logarithmic values. Error bars represent standard deviations from three measurements.

\section{Linearized MOPS Calibration Curves}

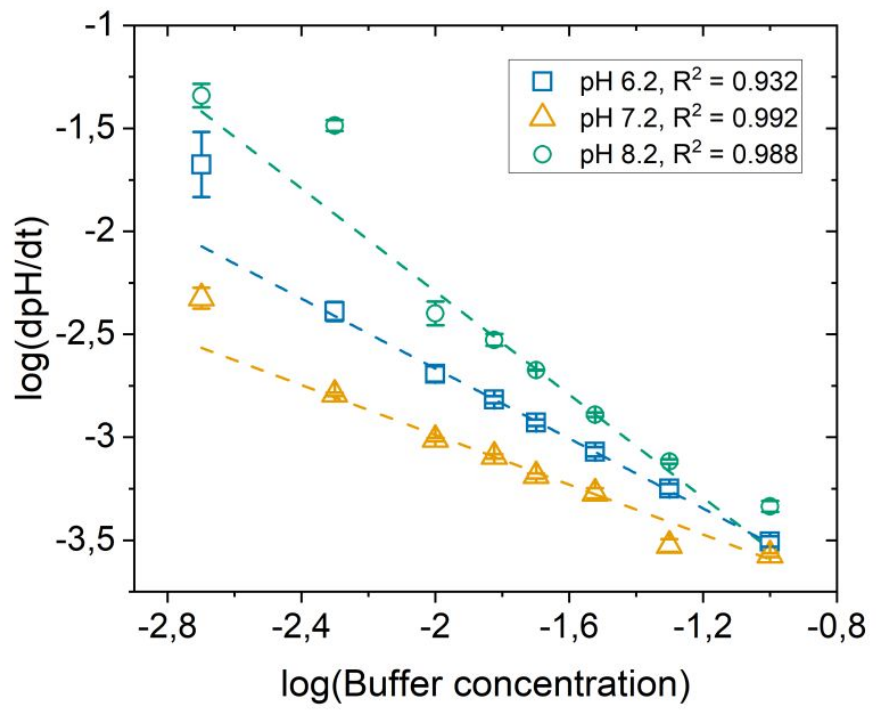

Figure S4. Linearized MOPS calibration curves at $\mathrm{pH} 6.2,7.2$ and 8.2 by double logarithmic representation of rate of $\mathrm{pH}$ change and buffer concentration. It has to be stated that these measurements were taken in a different measuring cell of similar design. Due to different diffusion lengths the measured values differ slightly from the calibration data shown in

Figure 3. Error bars represent standard deviations from three measurements. 


\section{Comparison between MOPS and HEPES Buffer}
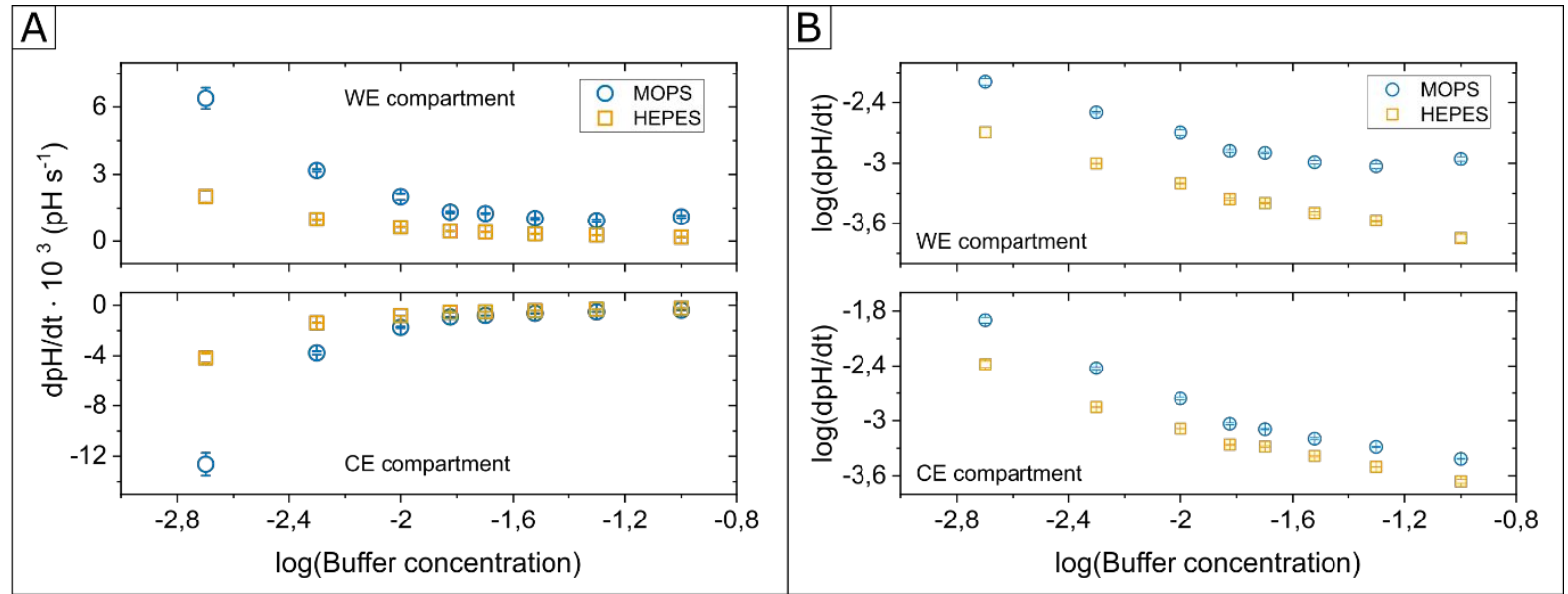

Figure S5. (A) Comparison of MOPS and HEPES calibration. MOPS generally shows bigger $\mathrm{pH}$ change rates in both directions due to higher conductivity of MOPS ions compared to HEPES ions. (B) Linearized calibrations by double logarithmic representation of rate of $\mathrm{pH}$ change and buffer concentration. For the linearization of the counter electrode compartment, absolute values for $\mathrm{dpH} / \mathrm{dt}$ were used to form logarithmic values. Error bars represent standard deviations from three measurements.
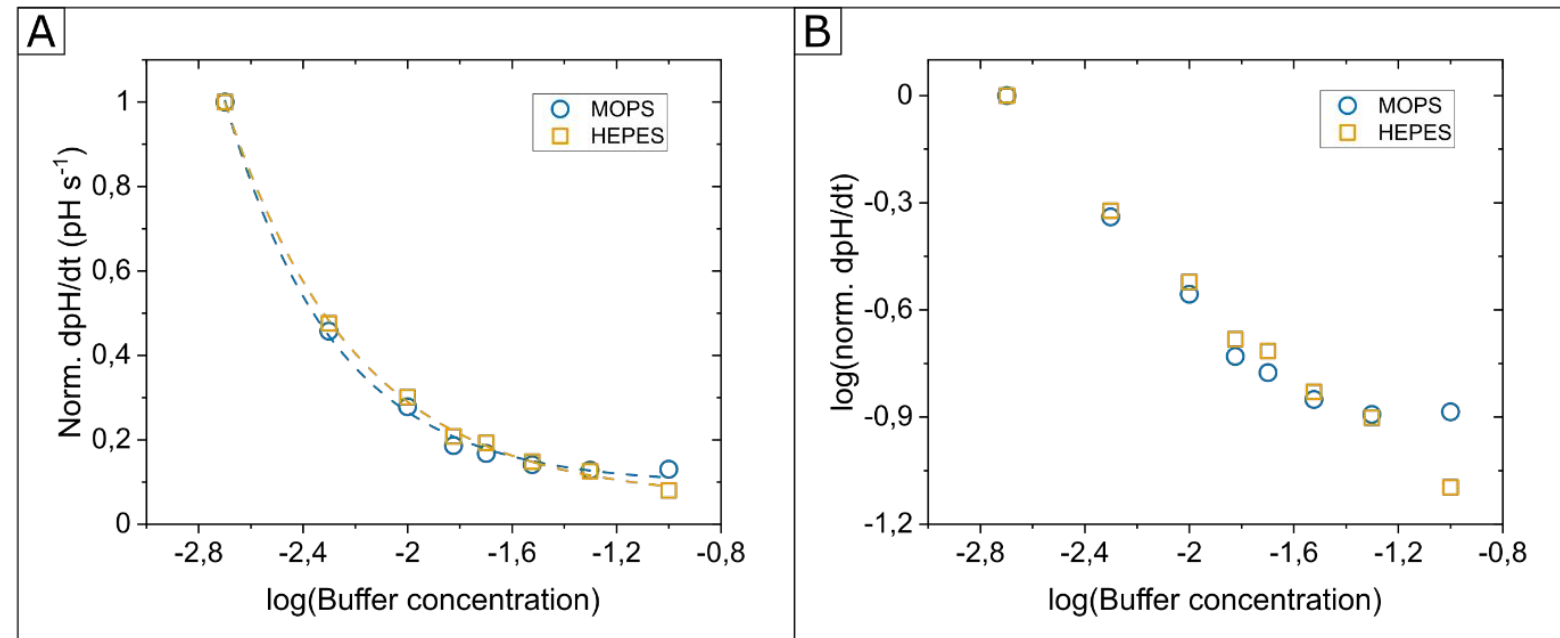

Figure S6. (A) Comparison of normalized pH change rate values of MOPS and HEPES calibration (working electrode). By normalizing the obtained values, the trend for both buffer species can be compared and seems to be congruent. (B) Linearized normalized calibrations of MOPS and HEPES by double logarithmic representation of normalized rate of $\mathrm{pH}$ change and buffer concentration. 


\section{Measurement Kinetics}

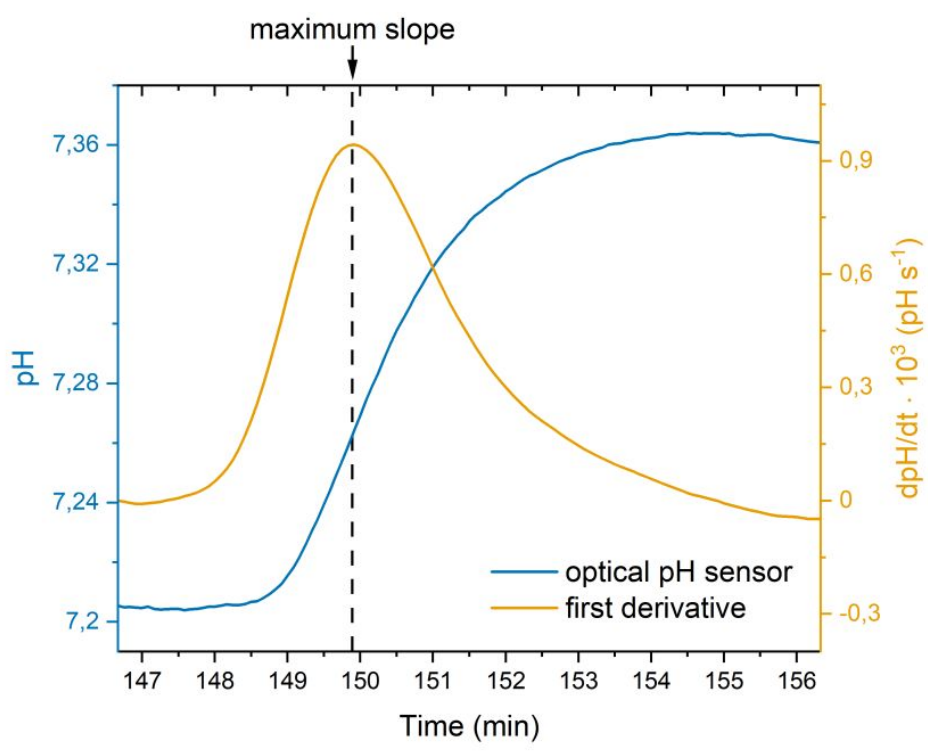

Figure S7. $\mathrm{pH}$ measurement and corresponding numerical first derivative $(\mathrm{dpH} / \mathrm{dt})$ of a single peak of the MOPS calibration ( $30 \mathrm{mM}$ is shown here). The maximum rate of $\mathrm{pH}$ change is reached after about 1 minute after the first $\mathrm{pH}$ change is observed.

\section{Comparison with pH Microelectrode}

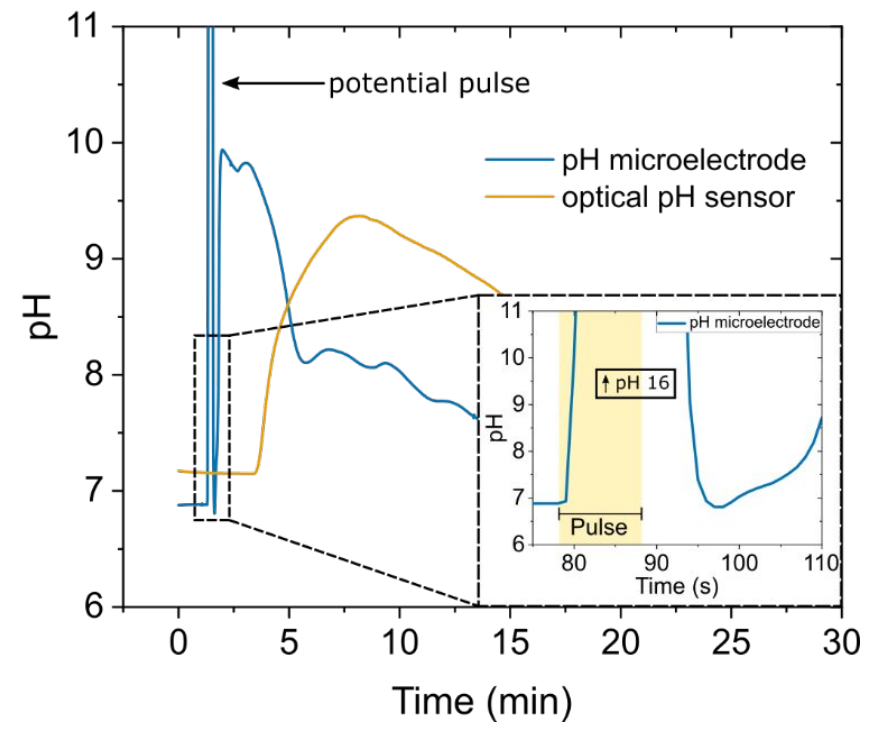

Figure S9. Comparison between optical $\mathrm{pH}$ sensor and potentiometric $\mathrm{pH}$ microsensor close to the working electrode. The experiment was performed in an open-cell setup, to facilitate the insertion of a $\mathrm{pH}$ microelectrode. A clear electrical interference can be seen at the time when the potential pulse is applied. It has to be stated that the optical sensor was placed at a $90^{\circ}$ angle a few millimetres away from the electrode, while the potentiometric microsensor was placed only a few micrometers next to the electrode. Differences in response time in this plot are therefore mainly governed by different diffusion lengths from the electrode to the respective sensor. 


\section{Simulation: 2D - proton diffusion in flow chamber $(t d s)$}

\section{Contents}

Contents......

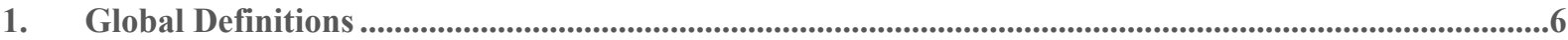

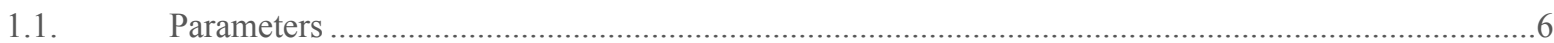

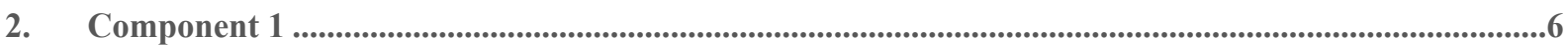

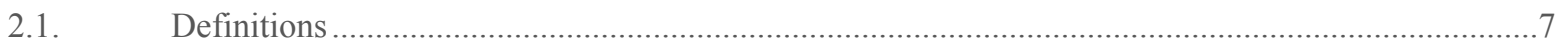

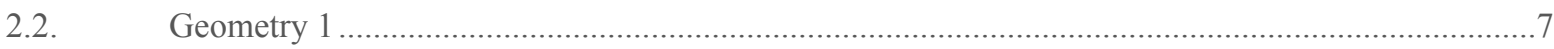

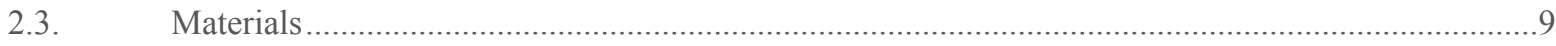

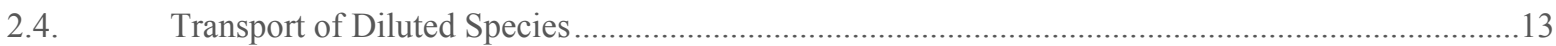

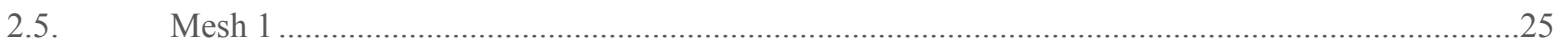

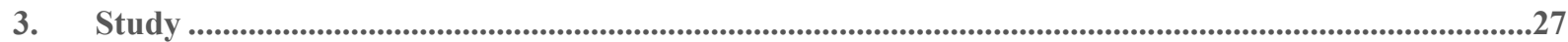

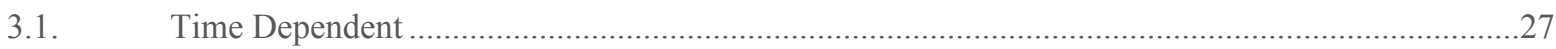

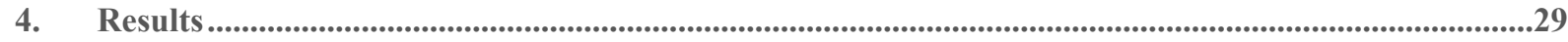

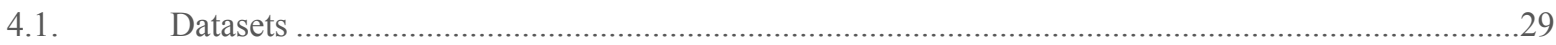

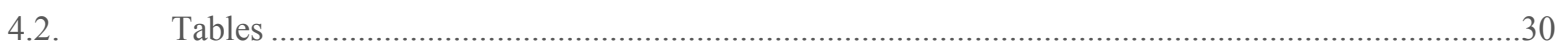

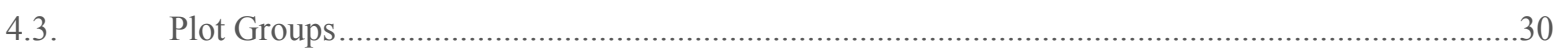




\section{Global Definitions}

Global settings

\begin{tabular}{|l|l|}
\hline Version & $\begin{array}{l}\text { COMSOL Multiphysics } 5.5 \text { (Build: } \\
306)\end{array}$ \\
\hline Unit system & SI \\
\hline
\end{tabular}

Used products

COMSOL Multiphysics

Chemical Reaction Engineering Module

\section{Parameters}

Parameters 1

\begin{tabular}{|l|l|l|l|}
\hline Name & Expression & Value & Description \\
\hline pKa & 7.13 & 7.13 & pka value buffer \\
\hline $\mathrm{Dp}$ & $1 \mathrm{e}-9\left[\mathrm{~m}^{\wedge} 2 / \mathrm{s}\right]$ & $1 \mathrm{E}-9 \mathrm{~m}^{2} / \mathrm{s}$ & diffusion coefficient proton \\
\hline $\mathrm{Db}$ & $2.10789 \mathrm{e}-11\left[\mathrm{~m}^{\wedge} 2 / \mathrm{s}\right]$ & $2.1079 \mathrm{E}-11 \mathrm{~m}^{2} / \mathrm{s}$ & diffusion coefficient buffer \\
\hline c_proton0 & $10 \mathrm{e}-7[\mathrm{~mol} / \mathrm{L}]$ & $0.001 \mathrm{~mol} / \mathrm{m}^{3}$ & concentration proton \\
\hline c_proton & $10^{\wedge}(-1)[\mathrm{mol} / \mathrm{L}]$ & $100 \mathrm{~mol} / \mathrm{m}^{3}$ & acid \\
\hline c_buffer & $15^{*} 10^{\wedge}(-3)[\mathrm{mol} / \mathrm{L}]$ & $15 \mathrm{~mol} / \mathrm{m}^{3}$ & concentration buffer \\
\hline
\end{tabular}

\section{Component 1}

Settings

\begin{tabular}{|l|l|}
\hline Description & Value \\
\hline Unit system & Same as global system \\
\hline Geometry shape order & Automatic \\
\hline
\end{tabular}

Spatial frame coordinates

\begin{tabular}{|l|l|l|}
\hline First & Second & Third \\
\hline $\mathrm{x}$ & $\mathrm{y}$ & $\mathrm{z}$ \\
\hline
\end{tabular}

Material frame coordinates

\begin{tabular}{|l|l|l|}
\hline First & Second & Third \\
\hline $\mathrm{X}$ & $\mathrm{Y}$ & $\mathrm{Z}$ \\
\hline
\end{tabular}

Geometry frame coordinates

\begin{tabular}{l|l|l|} 
First & Second & Third \\
\hline $\mathrm{Xg}$ & $\mathrm{Yg}$ & $\mathrm{Zg}$ \\
\hline
\end{tabular}

Mesh frame coordinates

\begin{tabular}{l|l|l|} 
First & Second & Third \\
\hline $\mathrm{Xm}$ & $\mathrm{Ym}$ & $\mathrm{Zm}$ \\
\hline
\end{tabular}




\section{Definitions}

\section{Functions}

$\mathrm{pH}$ value

\begin{tabular}{|l|l|}
\hline Function name & an1 \\
\hline Function type & Analytic
\end{tabular}

Definition

\begin{tabular}{|l|l|} 
Description & Value \\
\hline Expression & $\mathrm{pKa}-\log 10((\mathrm{cp}+\mathrm{cbuffer}) / \mathrm{cbuffer})$ \\
\hline Arguments & $\{\mathrm{cp}$, cbuffer $\}$
\end{tabular}

Coordinate Systems

Boundary System 1

\begin{tabular}{|l|l|}
\hline Coordinate system type & Boundary system \\
\hline Tag & sys1 \\
\hline
\end{tabular}

Coordinate names

\begin{tabular}{|l|l|l|}
\hline First & Second & Third \\
\hline t1 & $\mathrm{n}$ & to \\
\hline
\end{tabular}

\section{Geometry 1}

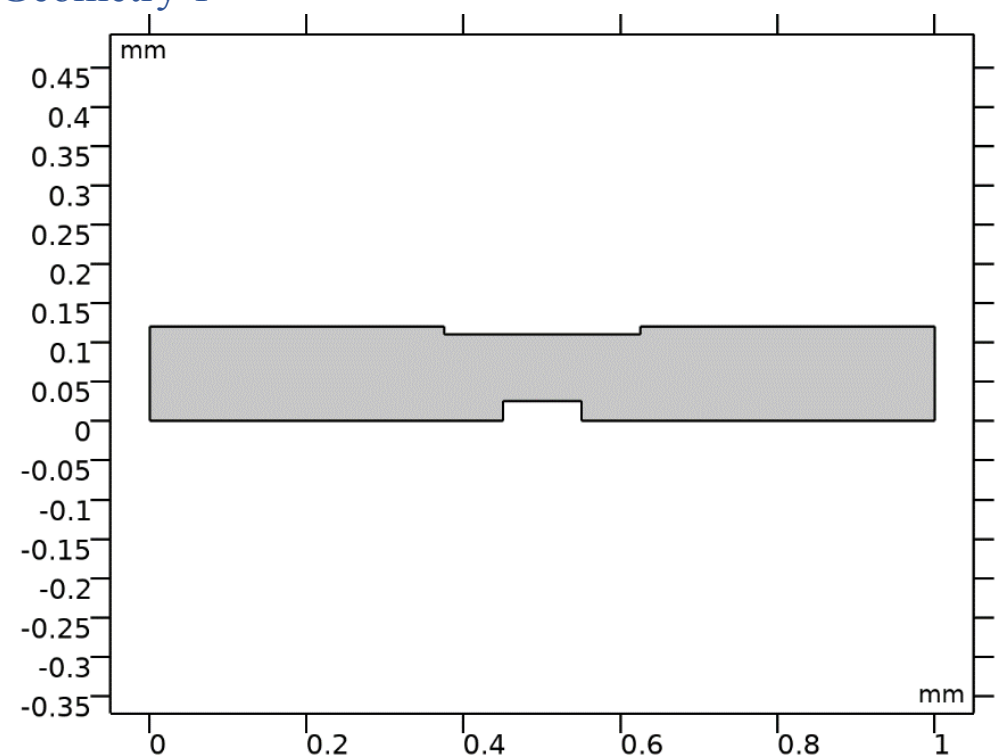

Geometry 1

Units

Length unit $\mathrm{mm}$

Angular unit deg

Geometry statistics

\begin{tabular}{|l|l|}
\hline Description & Value \\
\hline Space dimension & 2 \\
\hline
\end{tabular}




\begin{tabular}{|l|l|}
\hline Description & Value \\
\hline Number of domains & 1 \\
\hline Number of boundaries & 12 \\
\hline Number of vertices & 12 \\
\hline
\end{tabular}

Rectangle 1 (r1)

Position

\begin{tabular}{|l|l|}
\hline Description & Value \\
\hline Position & $\{0,0\}$ \\
\hline
\end{tabular}

Size

\begin{tabular}{|l|l|}
\hline Description & Value \\
\hline Width & 1 \\
\hline Height & 0.12 \\
\hline
\end{tabular}

Rectangle 2 (r2)

Position

\section{Description Value \\ Position $\{0.45,0\}$}

Size

\begin{tabular}{|l|l|}
\hline Description & Value \\
\hline Width & 0.1 \\
\hline Height & 0.025 \\
\hline
\end{tabular}

Rectangle 3 (r3)

Position

\begin{tabular}{|l|l|}
\hline Description & Value \\
\hline Position & $\{0.375,0.11\}$ \\
\hline
\end{tabular}

Size

\begin{tabular}{|l|l|}
\hline Description & Value \\
\hline Width & 0.25 \\
\hline Height & 0.015 \\
\hline
\end{tabular}




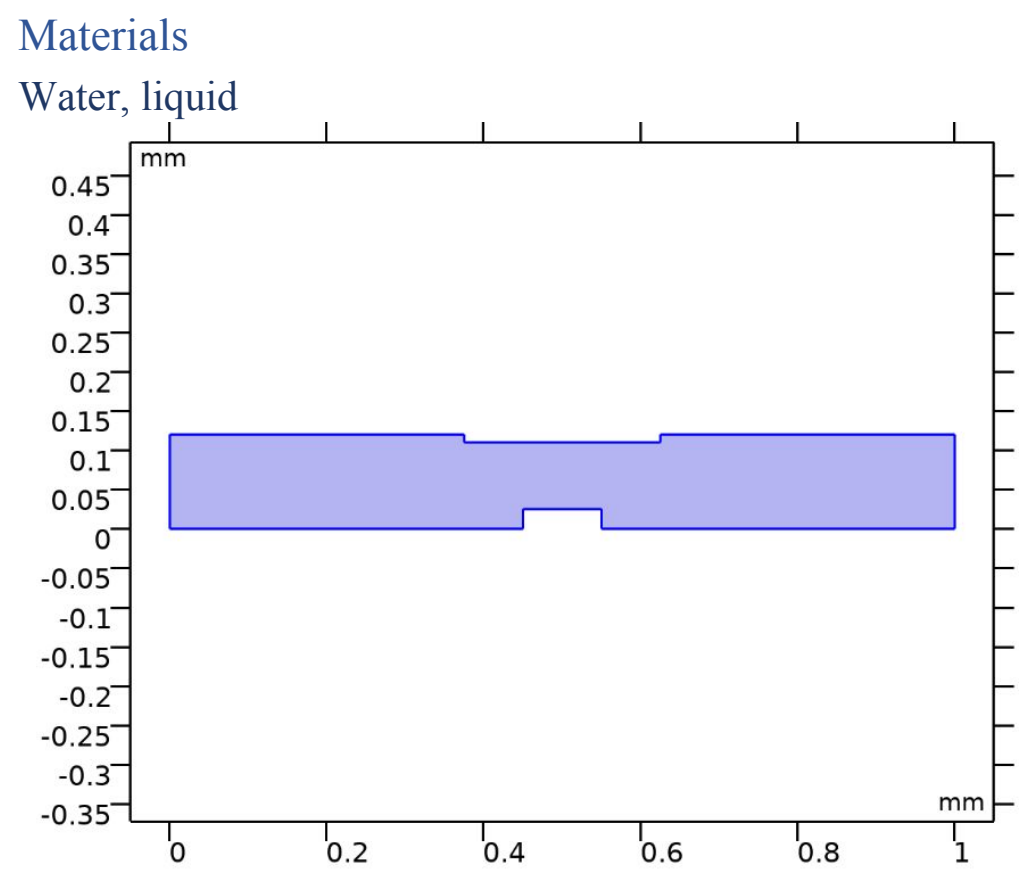

Water, liquid

\section{Selection}

\begin{tabular}{|l|l|}
\hline Geometric entity level & Domain \\
\hline Selection & Geometry geom1: Dimension 2: All domains \\
\hline
\end{tabular}

Basic Settings

\begin{tabular}{|c|c|}
\hline Description & Value \\
\hline Coefficient of thermal expansion & $\begin{array}{l}\{\{\text { alpha_p(T), } 0,0\},\{0, \text { alpha_p(T), } 0\},\{0,0, \\
\text { alpha_p(T) }\}\}\end{array}$ \\
\hline Bulk viscosity & $\operatorname{muB}(\mathrm{T})$ \\
\hline thermalexpansioncoefficient_symmetry & 3 \\
\hline bulkviscosity_symmetry & 0 \\
\hline Dynamic viscosity & $\operatorname{eta}(\mathrm{T})$ \\
\hline dynamicviscosity_symmetry & 0 \\
\hline Ratio of specific heats & gamma_w(T) \\
\hline ratioofspecificheat_symmetry & 0 \\
\hline Electrical conductivity & $\begin{array}{l}\{\{5.5 \mathrm{e}-6[\mathrm{~S} / \mathrm{m}], 0,0\},\{0,5.5 \mathrm{e}-6[\mathrm{~S} / \mathrm{m}], 0\},\{0,0,5.5 \mathrm{e}- \\
6[\mathrm{~S} / \mathrm{m}]\}\}\end{array}$ \\
\hline electricconductivity_symmetry & 3 \\
\hline Heat capacity at constant pressure & $\mathrm{Cp}(\mathrm{T})$ \\
\hline heatcapacity_symmetry & 0 \\
\hline Density & $\operatorname{rho}(\mathrm{T})$ \\
\hline density_symmetry & 0 \\
\hline Thermal conductivity & $\{\{\mathrm{k}(\mathrm{T}), 0,0\},\{0, \mathrm{k}(\mathrm{T}), 0\},\{0,0, \mathrm{k}(\mathrm{T})\}\}$ \\
\hline thermalconductivity_symmetry & 3 \\
\hline Speed of sound & $\operatorname{cs}(\mathrm{T})$ \\
\hline soundspeed_symmetry & 0 \\
\hline
\end{tabular}


Functions

\begin{tabular}{|l|l|}
\hline Function name & Type \\
\hline eta & Piecewise \\
\hline $\mathrm{Cp}$ & Piecewise \\
\hline rho & Piecewise \\
\hline $\mathrm{k}$ & Piecewise \\
\hline cs & Interpolation \\
\hline alpha_p & Analytic \\
\hline gamma_w & Analytic \\
\hline muB & Analytic \\
\hline
\end{tabular}

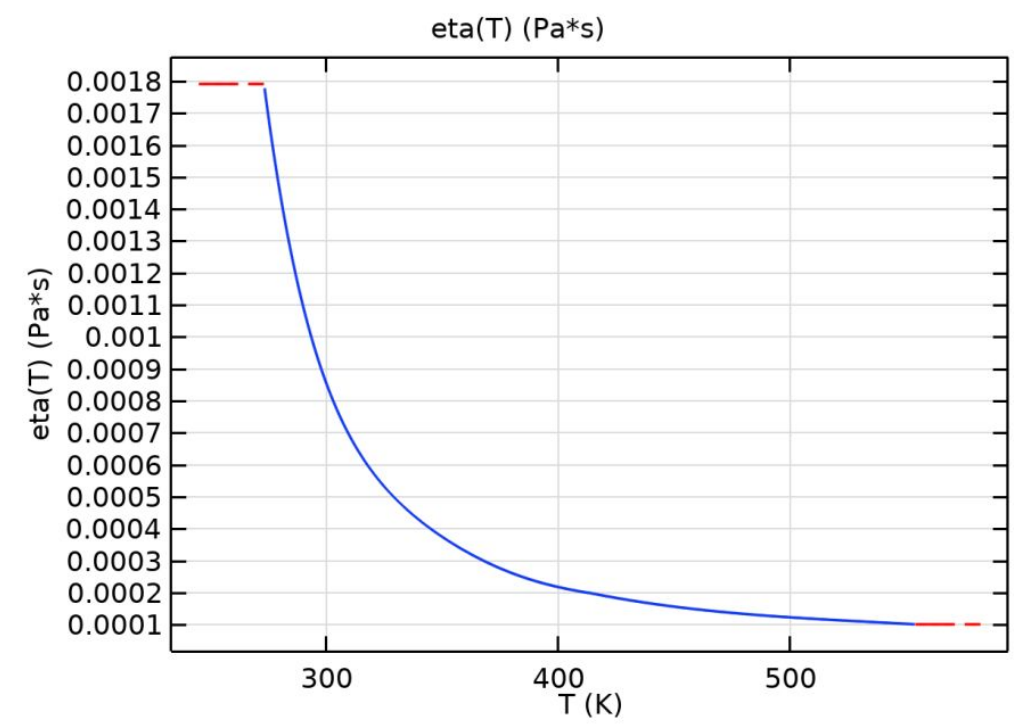

eta

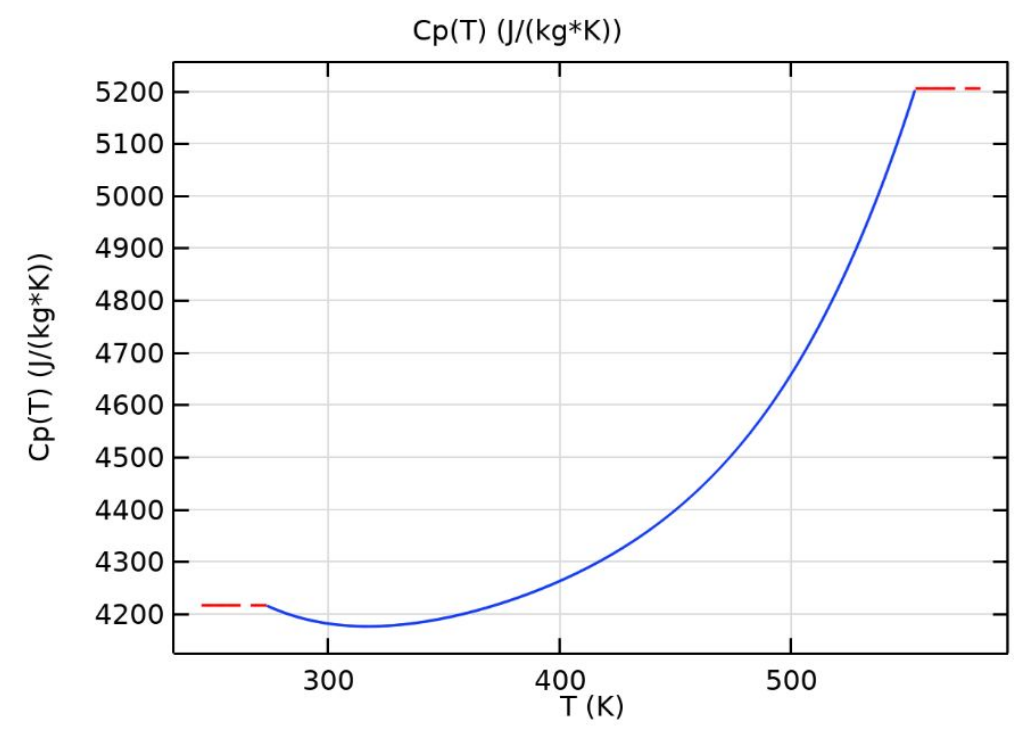

$C p$ 


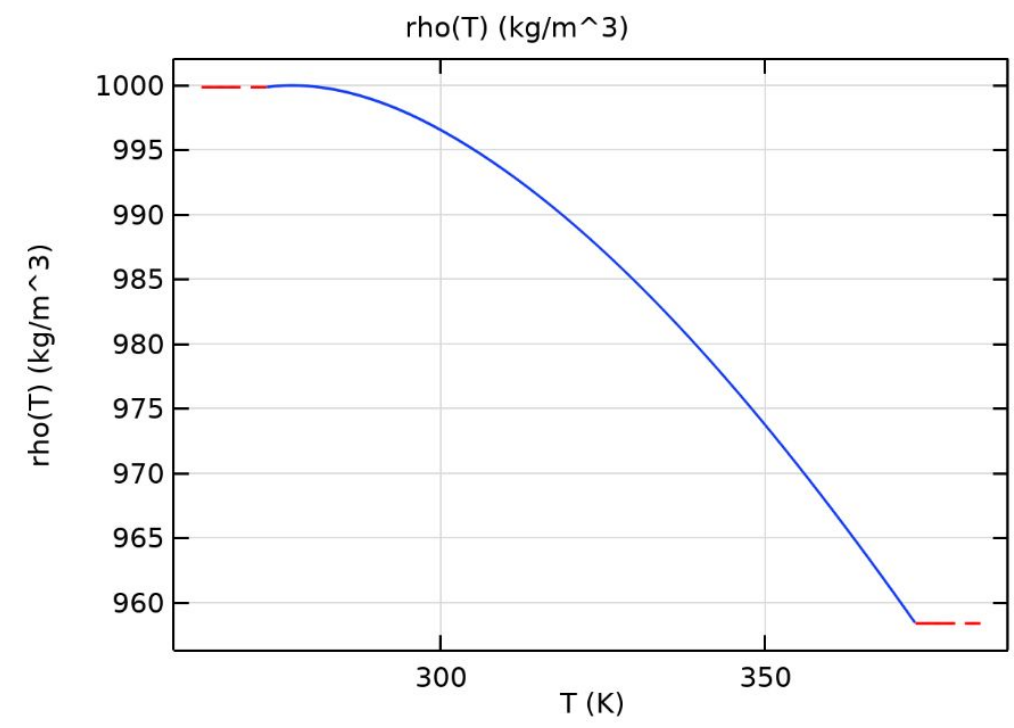

rho

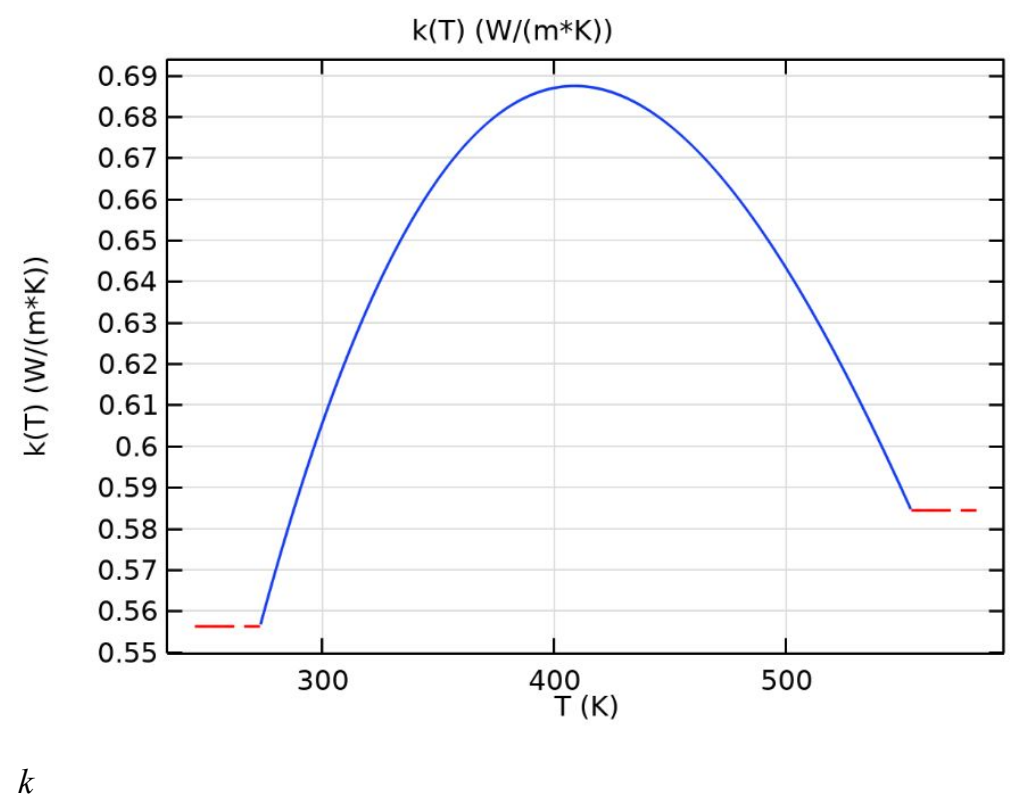




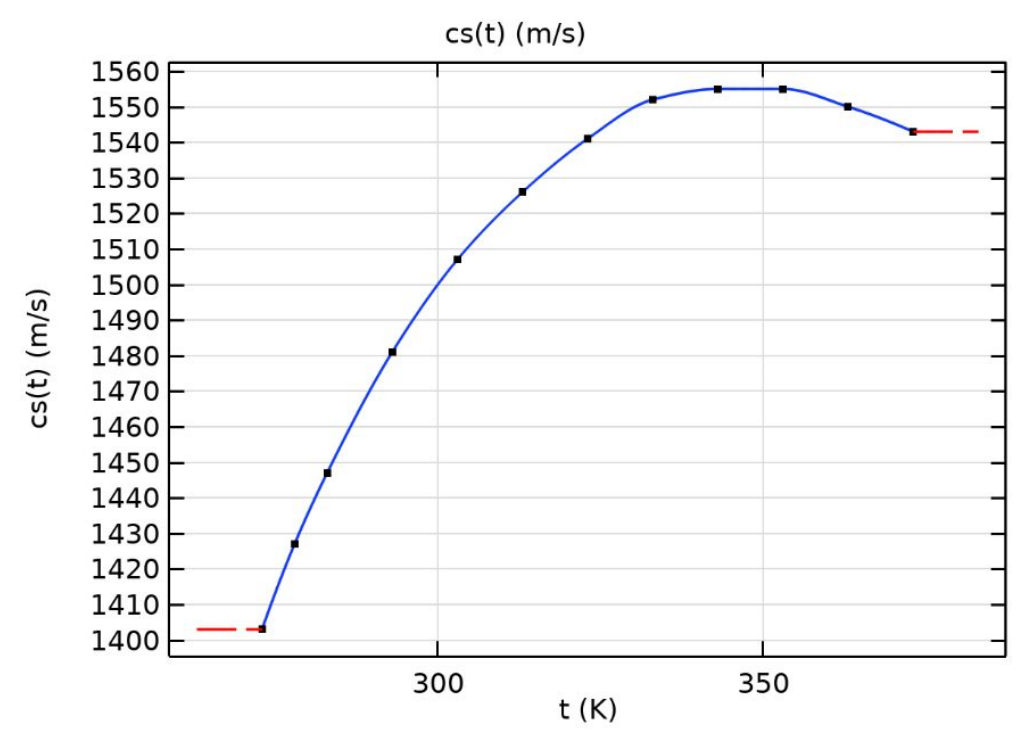

cs

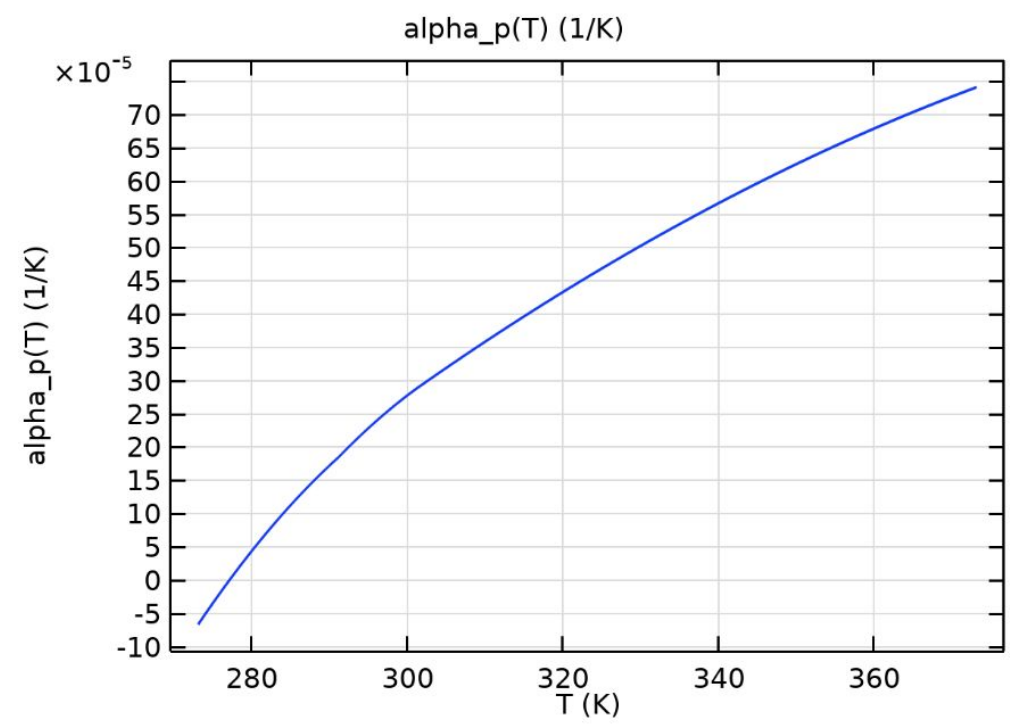

alpha_p 


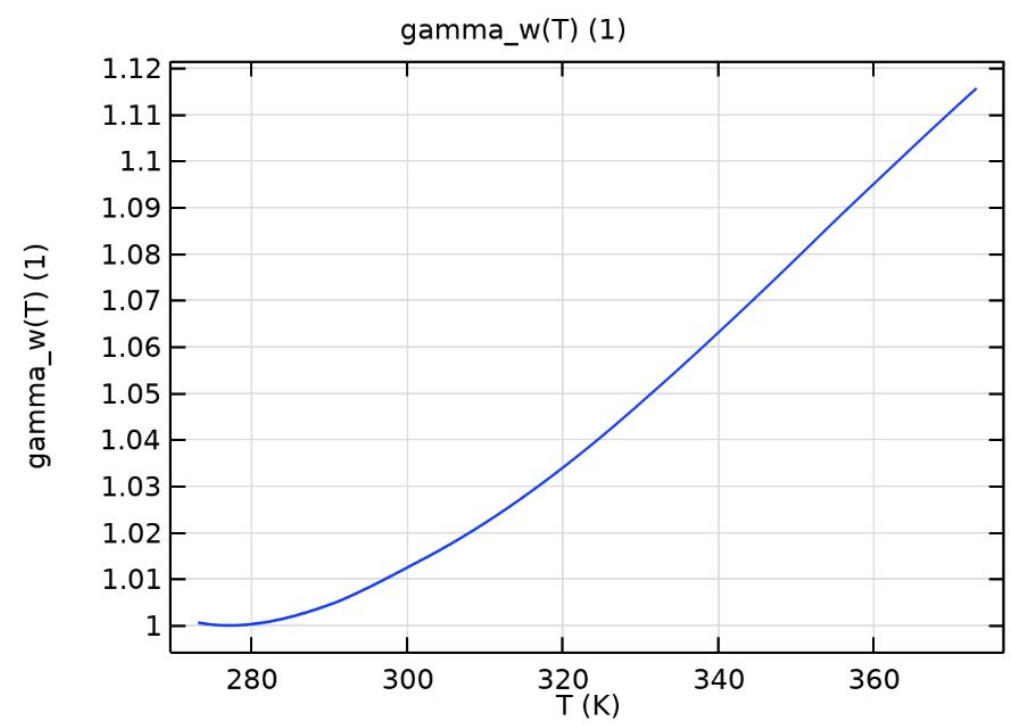

gamma_w

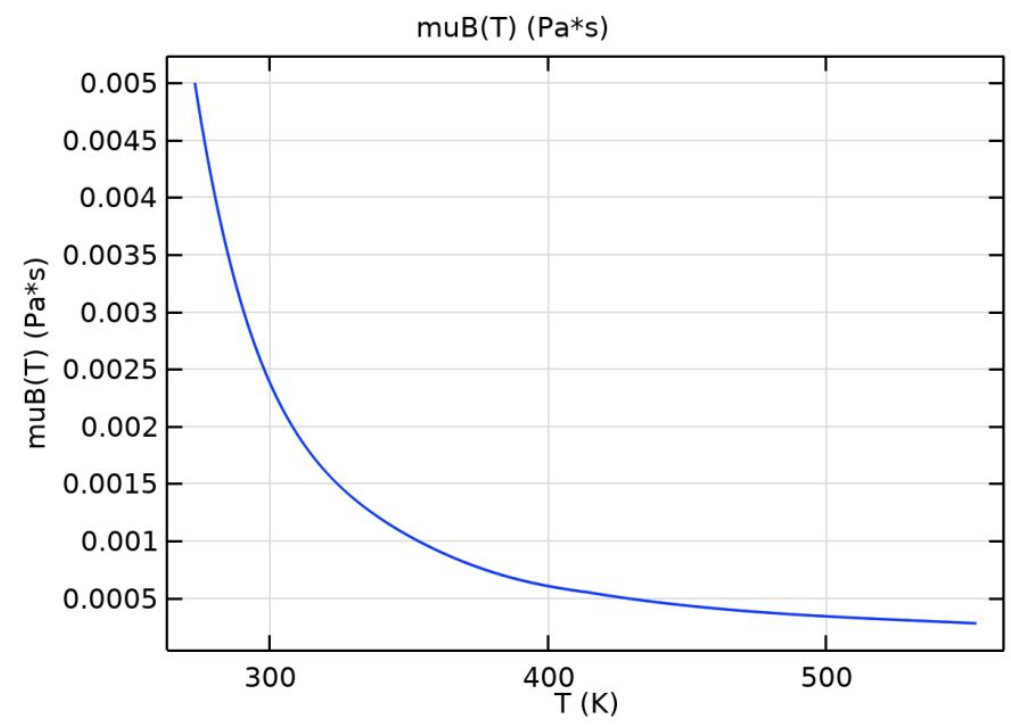

$m u B$

Transport of Diluted Species

Used products

COMSOL Multiphysics

Chemical Reaction Engineering Module 


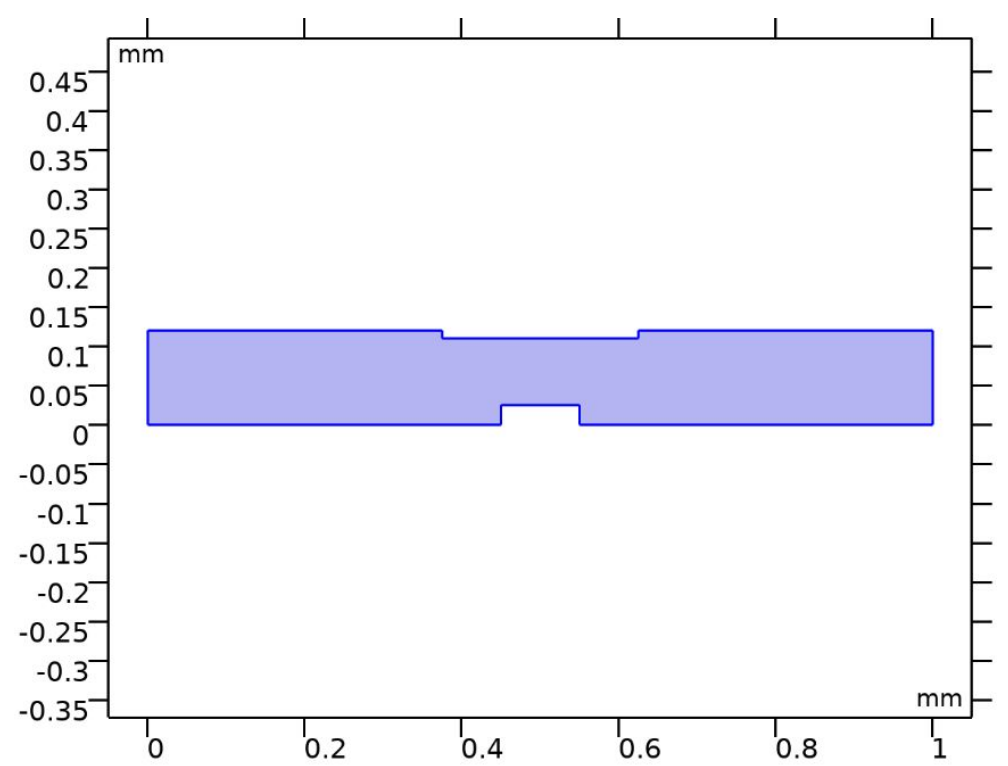

Transport of Diluted Species

\section{Selection}

Geometric entity level Domain

Selection Geometry geom1: Dimension 2: All domains

Equations

$\frac{\partial c_{i}}{\partial t}+\nabla \cdot \mathbf{J}_{i}+\mathbf{u} \cdot \nabla c_{i}=R_{i}$

$\mathbf{J}_{i}=-D_{i} \nabla c_{i}$

Interface settings

Discretization

Settings

\begin{tabular}{l|l|} 
Description & Value \\
\hline Concentration & Linear
\end{tabular}

Transport mechanisms

Settings

\begin{tabular}{|l|l|}
\hline Description & Value \\
\hline Convection & On \\
\hline Migration in electric field & Off \\
\hline Mass transfer in porous media & Off \\
\hline
\end{tabular}

Variables

\begin{tabular}{|l|l|l|l|l|l|}
\hline Name & Expression & Unit & Description & Selection & Details \\
\hline tds.R_cp & 0 & $\mathrm{~mol} /\left(\mathrm{m}^{3} \cdot \mathrm{s}\right)$ & $\begin{array}{l}\text { Total rate } \\
\text { expression }\end{array}$ & Domain 1 & + operation \\
\hline
\end{tabular}




\begin{tabular}{|c|c|c|c|c|c|}
\hline Name & Expression & Unit & Description & Selection & Details \\
\hline tds.cP_cp & 0 & $\mathrm{~mol} / \mathrm{kg}$ & $\begin{array}{l}\text { Concentration } \\
\text { species } \\
\text { absorbed to } \\
\text { the solid }\end{array}$ & Domain 1 & + operation \\
\hline tds.cP_cp & 0 & $\mathrm{~mol} / \mathrm{kg}$ & $\begin{array}{l}\text { Concentration } \\
\text { species } \\
\text { absorbed to } \\
\text { the solid }\end{array}$ & $\begin{array}{l}\text { Boundaries } \\
1-12\end{array}$ & + operation \\
\hline tds.KP_cp & 0 & $\mathrm{~m}^{3} / \mathrm{kg}$ & $\begin{array}{l}\text { Adsorption } \\
\text { isotherm, first } \\
\text { concentration } \\
\text { derivative }\end{array}$ & Domain 1 & + operation \\
\hline tds.KP_cp & 0 & $\mathrm{~m}^{3} / \mathrm{kg}$ & $\begin{array}{l}\text { Adsorption } \\
\text { isotherm, first } \\
\text { concentration } \\
\text { derivative }\end{array}$ & $\begin{array}{l}\text { Boundaries } \\
1-12\end{array}$ & + operation \\
\hline tds.R_cbuffer & 0 & $\mathrm{~mol} /\left(\mathrm{m}^{3} \cdot \mathrm{s}\right)$ & $\begin{array}{l}\text { Total rate } \\
\text { expression }\end{array}$ & Domain 1 & + operation \\
\hline tds.cP_cbuffer & 0 & $\mathrm{~mol} / \mathrm{kg}$ & $\begin{array}{l}\text { Concentration } \\
\text { species } \\
\text { absorbed to } \\
\text { the solid }\end{array}$ & Domain 1 & + operation \\
\hline tds.cP_cbuffer & 0 & $\mathrm{~mol} / \mathrm{kg}$ & $\begin{array}{l}\text { Concentration } \\
\text { species } \\
\text { absorbed to } \\
\text { the solid }\end{array}$ & $\begin{array}{l}\text { Boundaries } \\
1-12\end{array}$ & + operation \\
\hline tds.KP_cbuffer & 0 & $\mathrm{~m}^{3} / \mathrm{kg}$ & $\begin{array}{l}\text { Adsorption } \\
\text { isotherm, first } \\
\text { concentration } \\
\text { derivative }\end{array}$ & Domain 1 & + operation \\
\hline tds.KP_cbuffer & 0 & $\mathrm{~m}^{3} / \mathrm{kg}$ & $\begin{array}{l}\text { Adsorption } \\
\text { isotherm, first } \\
\text { concentration } \\
\text { derivative }\end{array}$ & $\begin{array}{l}\text { Boundaries } \\
1-12\end{array}$ & + operation \\
\hline tds.epsilon_p & 1 & 1 & Porosity & Domain 1 & \\
\hline tds.theta & tds.epsilon_p & 1 & $\begin{array}{l}\text { Liquid } \\
\text { volume } \\
\text { fraction }\end{array}$ & Domain 1 & \\
\hline tds.av & 0 & 1 & $\begin{array}{l}\text { Gas volume } \\
\text { fraction }\end{array}$ & Domain 1 & \\
\hline tds.nx & $\operatorname{dnx}$ & 1 & $\begin{array}{l}\text { Normal } \\
\text { vector, } \mathrm{x} \\
\text { component }\end{array}$ & $\begin{array}{l}\text { Boundaries } \\
1-12\end{array}$ & \\
\hline tds.ny & dny & 1 & $\begin{array}{l}\text { Normal } \\
\text { vector, y } \\
\text { component }\end{array}$ & $\begin{array}{l}\text { Boundaries } \\
1-12\end{array}$ & \\
\hline tds.nz & 0 & 1 & $\begin{array}{l}\text { Normal } \\
\text { vector, } \mathrm{z} \\
\text { component }\end{array}$ & $\begin{array}{l}\text { Boundaries } \\
1-12\end{array}$ & \\
\hline tds.nxmesh & dnxmesh & 1 & $\begin{array}{l}\text { Normal } \\
\text { vector } \\
\text { (mesh), x } \\
\text { component }\end{array}$ & $\begin{array}{l}\text { Boundaries } \\
1-12\end{array}$ & \\
\hline
\end{tabular}




\begin{tabular}{|c|c|c|c|c|c|}
\hline Name & Expression & Unit & Description & Selection & Details \\
\hline tds.nymesh & dnymesh & 1 & $\begin{array}{l}\text { Normal } \\
\text { vector } \\
\text { (mesh), y } \\
\text { component }\end{array}$ & $\begin{array}{l}\text { Boundaries } \\
1-12\end{array}$ & \\
\hline tds.nzmesh & 0 & 1 & $\begin{array}{l}\text { Normal } \\
\text { vector } \\
\text { (mesh), z } \\
\text { component }\end{array}$ & $\begin{array}{l}\text { Boundaries } \\
1-12\end{array}$ & \\
\hline tds.nxc & root.nxc/tds.ncLen & 1 & $\begin{array}{l}\text { Normal } \\
\text { vector, } \mathrm{x} \\
\text { component }\end{array}$ & $\begin{array}{l}\text { Boundaries } \\
1-12\end{array}$ & \\
\hline tds.nyc & root.nyc/tds.ncLen & 1 & $\begin{array}{l}\text { Normal } \\
\text { vector, y } \\
\text { component }\end{array}$ & $\begin{array}{l}\text { Boundaries } \\
1-12\end{array}$ & \\
\hline tds.nzc & 0 & 1 & $\begin{array}{l}\text { Normal } \\
\text { vector, z } \\
\text { component }\end{array}$ & $\begin{array}{l}\text { Boundaries } \\
1-12\end{array}$ & \\
\hline tds.ncLen & $\begin{array}{l}\text { sqrt(root.nxc }{ }^{\wedge} 2+ \\
\text { root.nyc } 2+\text { eps })\end{array}$ & 1 & Help variable & $\begin{array}{l}\text { Boundaries } \\
1-12\end{array}$ & \\
\hline tds.cbf_cp & 0 & $\mathrm{~mol} /\left(\mathrm{m}^{2} \cdot \mathrm{s}\right)$ & $\begin{array}{l}\text { Convective } \\
\text { boundary flux }\end{array}$ & $\begin{array}{l}\text { Boundaries } \\
1-12\end{array}$ & \\
\hline tds.u & 0 & $\mathrm{~m} / \mathrm{s}$ & $\begin{array}{l}\text { Velocity } \\
\text { field, } x \\
\text { component }\end{array}$ & Domain 1 & \\
\hline tds.v & 0 & $\mathrm{~m} / \mathrm{s}$ & $\begin{array}{l}\text { Velocity } \\
\text { field, y } \\
\text { component }\end{array}$ & Domain 1 & \\
\hline tds.w & 0 & $\mathrm{~m} / \mathrm{s}$ & $\begin{array}{l}\text { Velocity } \\
\text { field, z } \\
\text { component }\end{array}$ & Domain 1 & \\
\hline tds.cbf_cbuffer & 0 & $\mathrm{~mol} /\left(\mathrm{m}^{2} \cdot \mathrm{s}\right)$ & $\begin{array}{l}\text { Convective } \\
\text { boundary flux }\end{array}$ & $\begin{array}{l}\text { Boundaries } \\
1-12\end{array}$ & \\
\hline
\end{tabular}

\section{Transport Properties 1}

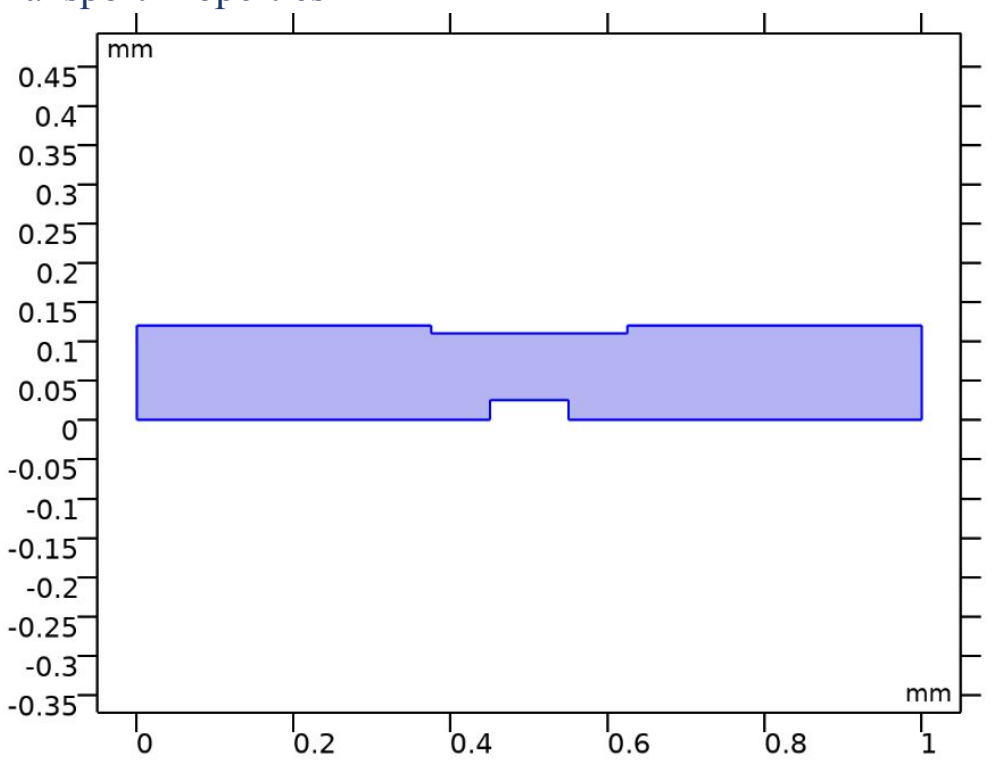

Transport Properties 1 


\section{Selection}

\begin{tabular}{l|l} 
Geometric entity level & Domain \\
\hline Selection & Geometry geom1: Dimension 2: All domains
\end{tabular}

Equations

$$
\begin{aligned}
& \frac{\partial c_{i}}{\partial t}+\nabla \cdot \mathbf{j}_{i}+\mathbf{u} \cdot \nabla c_{i}=R_{i} \\
& \underset{\mathbf{J}_{i}=-D_{i} \nabla c_{i}}{ }-\cdots \cdots \cdots \cdots \\
&
\end{aligned}
$$

\section{Convection}

Settings

\begin{tabular}{|l|l|}
\hline Description & Value \\
\hline Velocity field & User defined \\
\hline Velocity field & $\{0,0,0\}$ \\
\hline
\end{tabular}

Diffusion

Settings

\begin{tabular}{|l|l|}
\hline Description & Value \\
\hline Material & Water, liquid (mat 1$)$ \\
\hline Diffusion coefficient & User defined \\
\hline Diffusion coefficient & $\{\{\mathrm{Dp}, 0,0\},\{0, \mathrm{Dp}, 0\},\{0,0, \mathrm{Dp}\}\}$ \\
\hline Diffusion coefficient & User defined \\
\hline Diffusion coefficient & $\{\{\mathrm{Db}, 0,0\},\{0, \mathrm{Db}, 0\},\{0,0, \mathrm{Db}\}\}$ \\
\hline
\end{tabular}

Coordinate system selection

Settings

\begin{tabular}{|l|l|}
\hline Description & Value \\
\hline Coordinate system & Global coordinate system \\
\hline
\end{tabular}

Model input

Settings

\begin{tabular}{|l|l|}
\hline Description & Value \\
\hline Temperature & Common model input \\
\hline
\end{tabular}

\begin{tabular}{|c|c|c|c|c|c|}
\hline Name & Expression & Unit & Description & Selection & Details \\
\hline $\begin{array}{l}\text { domflux.cp } \\
\mathrm{x}\end{array}$ & tds.dflux_cpx & $\mathrm{mol} /\left(\mathrm{m}^{2} \cdot \mathrm{s}\right)$ & $\begin{array}{l}\text { Domain flux, } \mathrm{x} \\
\text { component }\end{array}$ & Domain 1 & \\
\hline $\begin{array}{l}\text { domflux.cp } \\
\text { y }\end{array}$ & tds.dflux_cpy & $\mathrm{mol} /\left(\mathrm{m}^{2} \cdot \mathrm{s}\right)$ & $\begin{array}{l}\text { Domain flux, y } \\
\text { component }\end{array}$ & Domain 1 & \\
\hline $\begin{array}{l}\text { domflux.cb } \\
\text { ufferx }\end{array}$ & tds.dflux_cbufferx & $\mathrm{mol} /\left(\mathrm{m}^{2} \cdot \mathrm{s}\right)$ & $\begin{array}{l}\text { Domain flux, } \mathrm{x} \\
\text { component }\end{array}$ & Domain 1 & \\
\hline $\begin{array}{l}\text { domflux.cb } \\
\text { uffery }\end{array}$ & tds.dflux_cbuffery & $\mathrm{mol} /\left(\mathrm{m}^{2} \cdot \mathrm{s}\right)$ & $\begin{array}{l}\text { Domain flux, y } \\
\text { component }\end{array}$ & Domain 1 & \\
\hline $\begin{array}{l}\text { tds.ndflux } \\
\text { cp }\end{array}$ & tds.bndFlux_cp & $\mathrm{mol} /\left(\mathrm{m}^{2} \cdot \mathrm{s}\right)$ & $\begin{array}{l}\text { Normal } \\
\text { diffusive flux }\end{array}$ & $\begin{array}{l}\text { Boundaries } \\
1-12\end{array}$ & \\
\hline
\end{tabular}

Variables 


\begin{tabular}{|c|c|c|c|c|c|}
\hline Name & Expression & Unit & Description & Selection & Details \\
\hline $\begin{array}{l}\text { tds.ncflux_- } \\
\mathrm{cp}\end{array}$ & $\begin{array}{l}\text { tds.cflux_cpx*tds.nxc+tds.cflux } \\
\text { cpy*tds.nyc+tds.cflux_cpz*td } \\
\text { s.nzc }\end{array}$ & $\mathrm{mol} /\left(\mathrm{m}^{2} \cdot \mathrm{s}\right)$ & $\begin{array}{l}\text { Normal } \\
\text { convective flux }\end{array}$ & $\begin{array}{l}\text { Boundaries } \\
1-12\end{array}$ & \\
\hline $\begin{array}{l}\text { tds.ntflux_c } \\
\mathrm{p}\end{array}$ & $\begin{array}{l}\text { tds.bndFlux_cp+tds.cflux_cpx* } \\
\text { tds.nxc+tds.cflux_cpy*tds.nyc+ } \\
\text { tds.cflux_cpz*tds.nzc }\end{array}$ & $\mathrm{mol} /\left(\mathrm{m}^{2} \cdot \mathrm{s}\right)$ & $\begin{array}{l}\text { Normal total } \\
\text { flux }\end{array}$ & $\begin{array}{l}\text { Boundaries } \\
1-12\end{array}$ & \\
\hline $\begin{array}{l}\text { tds.ndflux_- } \\
\text { cbuffer }\end{array}$ & tds.bndFlux_cbuffer & $\mathrm{mol} /\left(\mathrm{m}^{2} \cdot \mathrm{s}\right)$ & $\begin{array}{l}\text { Normal } \\
\text { diffusive flux }\end{array}$ & $\begin{array}{l}\text { Boundaries } \\
1-12\end{array}$ & \\
\hline $\begin{array}{l}\text { tds.ncflux } \\
\text { cbuffer }\end{array}$ & $\begin{array}{l}\text { tds.cflux_cbufferx } * \text { tds.nxc+tds. } \\
\text { cflux_cbuffery*tds.nyc+tds.cfl } \\
\text { ux_cbufferz*tds.nzc }\end{array}$ & $\mathrm{mol} /\left(\mathrm{m}^{2} \cdot \mathrm{s}\right)$ & $\begin{array}{l}\text { Normal } \\
\text { convective flux }\end{array}$ & $\begin{array}{l}\text { Boundaries } \\
1-12\end{array}$ & \\
\hline $\begin{array}{l}\text { tds.ntflux_c } \\
\text { buffer }\end{array}$ & $\begin{array}{l}\text { tds.bndFlux_cbuffer+tds.cflux_- } \\
\text { cbufferx*tds.nxc+tds.cflux_cbu } \\
\text { ffery*tds.nyc+tds.cflux_cbuffer } \\
\text { z*tds.nzc }\end{array}$ & $\mathrm{mol} /\left(\mathrm{m}^{2} \cdot \mathrm{s}\right)$ & $\begin{array}{l}\text { Normal total } \\
\text { flux }\end{array}$ & $\begin{array}{l}\text { Boundaries } \\
1-12\end{array}$ & \\
\hline tds.u & model.input.u1 & $\mathrm{m} / \mathrm{s}$ & $\begin{array}{l}\text { Velocity field, } x \\
\text { component }\end{array}$ & Domain 1 & Meta \\
\hline tds.v & model.input.u2 & $\mathrm{m} / \mathrm{s}$ & $\begin{array}{l}\text { Velocity field, y } \\
\text { component }\end{array}$ & Domain 1 & Meta \\
\hline tds.w & model.input.u3 & $\mathrm{m} / \mathrm{s}$ & $\begin{array}{l}\text { Velocity field, z } \\
\text { component }\end{array}$ & Domain 1 & Meta \\
\hline tds.D_cpxx & $\mathrm{Dp}$ & $\mathrm{m}^{2} / \mathrm{s}$ & $\begin{array}{l}\text { Diffusion } \\
\text { coefficient, } \mathrm{xx} \\
\text { component }\end{array}$ & Domain 1 & $\begin{array}{l}+ \text { operati } \\
\text { on }\end{array}$ \\
\hline tds.D_cpyx & 0 & $\mathrm{~m}^{2} / \mathrm{s}$ & $\begin{array}{l}\text { Diffusion } \\
\text { coefficient, yx } \\
\text { component }\end{array}$ & Domain 1 & $\begin{array}{l}+ \text { operati } \\
\text { on }\end{array}$ \\
\hline tds.D_cpzx & 0 & $\mathrm{~m}^{2} / \mathrm{s}$ & $\begin{array}{l}\text { Diffusion } \\
\text { coefficient, zx } \\
\text { component }\end{array}$ & Domain 1 & $\begin{array}{l}+ \text { operati } \\
\text { on }\end{array}$ \\
\hline tds.D_cpxy & 0 & $\mathrm{~m}^{2} / \mathrm{s}$ & $\begin{array}{l}\text { Diffusion } \\
\text { coefficient, xy } \\
\text { component }\end{array}$ & Domain 1 & $\begin{array}{l}+ \text { operati } \\
\text { on }\end{array}$ \\
\hline tds.D_cpyy & $\mathrm{Dp}$ & $\mathrm{m}^{2} / \mathrm{s}$ & $\begin{array}{l}\text { Diffusion } \\
\text { coefficient, yy } \\
\text { component }\end{array}$ & Domain 1 & $\begin{array}{l}+ \text { operati } \\
\text { on }\end{array}$ \\
\hline tds.D_cpzy & 0 & $\mathrm{~m}^{2} / \mathrm{s}$ & $\begin{array}{l}\text { Diffusion } \\
\text { coefficient, zy } \\
\text { component }\end{array}$ & Domain 1 & $\begin{array}{l}+ \text { operati } \\
\text { on }\end{array}$ \\
\hline tds.D_cpxz & 0 & $\mathrm{~m}^{2} / \mathrm{s}$ & $\begin{array}{l}\text { Diffusion } \\
\text { coefficient, } \mathrm{xz} \\
\text { component }\end{array}$ & Domain 1 & $\begin{array}{l}+ \text { operati } \\
\text { on }\end{array}$ \\
\hline tds.D_cpyz & 0 & $\mathrm{~m}^{2} / \mathrm{s}$ & $\begin{array}{l}\text { Diffusion } \\
\text { coefficient, yz } \\
\text { component }\end{array}$ & Domain 1 & $\begin{array}{l}+ \text { operati } \\
\text { on }\end{array}$ \\
\hline tds.D_cpzz & $\mathrm{Dp}$ & $\mathrm{m}^{2} / \mathrm{s}$ & $\begin{array}{l}\text { Diffusion } \\
\text { coefficient, zz } \\
\text { component }\end{array}$ & Domain 1 & $\begin{array}{l}+ \text { operati } \\
\text { on }\end{array}$ \\
\hline $\begin{array}{l}\text { tds.D_cbuff } \\
\text { erxx }\end{array}$ & $\mathrm{Db}$ & $\mathrm{m}^{2} / \mathrm{s}$ & $\begin{array}{l}\text { Diffusion } \\
\text { coefficient, } \mathrm{xx} \\
\text { component }\end{array}$ & Domain 1 & $\begin{array}{l}+ \text { operati } \\
\text { on }\end{array}$ \\
\hline
\end{tabular}




\begin{tabular}{|c|c|c|c|c|c|}
\hline Name & Expression & Unit & Description & Selection & Details \\
\hline $\begin{array}{l}\text { tds.D_cbuff } \\
\text { eryx }\end{array}$ & 0 & $\mathrm{~m}^{2} / \mathrm{s}$ & $\begin{array}{l}\text { Diffusion } \\
\text { coefficient, yx } \\
\text { component }\end{array}$ & Domain 1 & $\begin{array}{l}+ \text { operati } \\
\text { on }\end{array}$ \\
\hline $\begin{array}{l}\text { tds.D_cbuff } \\
\text { erzx }\end{array}$ & 0 & $\mathrm{~m}^{2} / \mathrm{s}$ & $\begin{array}{l}\text { Diffusion } \\
\text { coefficient, zx } \\
\text { component }\end{array}$ & Domain 1 & $\begin{array}{l}+ \text { operati } \\
\text { on }\end{array}$ \\
\hline $\begin{array}{l}\text { tds.D_cbuff } \\
\text { erxy }\end{array}$ & 0 & $\mathrm{~m}^{2} / \mathrm{s}$ & $\begin{array}{l}\text { Diffusion } \\
\text { coefficient, xy } \\
\text { component }\end{array}$ & Domain 1 & $\begin{array}{l}+ \text { operati } \\
\text { on }\end{array}$ \\
\hline $\begin{array}{l}\text { tds.D_cbuff } \\
\text { eryy }\end{array}$ & $\mathrm{Db}$ & $\mathrm{m}^{2} / \mathrm{s}$ & $\begin{array}{l}\text { Diffusion } \\
\text { coefficient, yy } \\
\text { component }\end{array}$ & Domain 1 & $\begin{array}{l}+ \text { operati } \\
\text { on }\end{array}$ \\
\hline $\begin{array}{l}\text { tds.D_cbuff } \\
\text { erzy }\end{array}$ & 0 & $\mathrm{~m}^{2} / \mathrm{s}$ & $\begin{array}{l}\text { Diffusion } \\
\text { coefficient, zy } \\
\text { component }\end{array}$ & Domain 1 & $\begin{array}{l}+ \text { operati } \\
\text { on }\end{array}$ \\
\hline $\begin{array}{l}\text { tds.D_cbuff } \\
\text { erxz }\end{array}$ & 0 & $\mathrm{~m}^{2} / \mathrm{s}$ & $\begin{array}{l}\text { Diffusion } \\
\text { coefficient, } x z \\
\text { component }\end{array}$ & Domain 1 & $\begin{array}{l}+ \text { operati } \\
\text { on }\end{array}$ \\
\hline $\begin{array}{l}\text { tds.D_cbuff } \\
\text { eryz }\end{array}$ & 0 & $\mathrm{~m}^{2} / \mathrm{s}$ & $\begin{array}{l}\text { Diffusion } \\
\text { coefficient, yz } \\
\text { component }\end{array}$ & Domain 1 & $\begin{array}{l}+ \text { operati } \\
\text { on }\end{array}$ \\
\hline $\begin{array}{l}\text { tds.D_cbuff } \\
\text { erzz }\end{array}$ & $\mathrm{Db}$ & $\mathrm{m}^{2} / \mathrm{s}$ & $\begin{array}{l}\text { Diffusion } \\
\text { coefficient, zz } \\
\text { component }\end{array}$ & Domain 1 & $\begin{array}{l}+ \text { operati } \\
\text { on }\end{array}$ \\
\hline tds.Dav_cp & $0.5 *\left(t d s . D \_c p x x+t d s . D \_c p y y\right)$ & $\mathrm{m}^{2} / \mathrm{s}$ & $\begin{array}{l}\text { Average } \\
\text { diffusion } \\
\text { coefficient }\end{array}$ & Domain 1 & \\
\hline $\begin{array}{l}\text { tds.Dav_cb } \\
\text { uffer }\end{array}$ & $\begin{array}{l}0.5 * \text { (tds.D_cbufferxx }+ \text { tds.D_cb } \\
\text { ufferyy) }\end{array}$ & $\mathrm{m}^{2} / \mathrm{s}$ & $\begin{array}{l}\text { Average } \\
\text { diffusion } \\
\text { coefficient }\end{array}$ & Domain 1 & \\
\hline $\begin{array}{l}\text { tds.tflux_cp } \\
\mathrm{x}\end{array}$ & tds.dflux_cpx+tds.cflux_cpx & $\mathrm{mol} /\left(\mathrm{m}^{2} \cdot \mathrm{s}\right)$ & $\begin{array}{l}\text { Total flux, } x \\
\text { component }\end{array}$ & Domain 1 & $\begin{array}{l}+ \text { operati } \\
\text { on }\end{array}$ \\
\hline $\begin{array}{l}\text { tds.tflux_cp } \\
\mathrm{y}\end{array}$ & tds.dflux_cpy+tds.cflux_cpy & $\mathrm{mol} /\left(\mathrm{m}^{2} \cdot \mathrm{s}\right)$ & $\begin{array}{l}\text { Total flux, y } \\
\text { component }\end{array}$ & Domain 1 & $\begin{array}{l}+ \text { operati } \\
\text { on }\end{array}$ \\
\hline $\begin{array}{l}\text { tds.tflux_cp } \\
\text { z }\end{array}$ & tds.dflux_cpz+tds.cflux_cpz & $\mathrm{mol} /\left(\mathrm{m}^{2} \cdot \mathrm{s}\right)$ & $\begin{array}{l}\text { Total flux, z } \\
\text { component }\end{array}$ & Domain 1 & $\begin{array}{l}+ \text { operati } \\
\text { on }\end{array}$ \\
\hline $\begin{array}{l}\text { tds.dfluxM } \\
\text { ag_cp }\end{array}$ & $\begin{array}{l}\text { sqrt(tds.dflux_cpx }{ }^{\wedge} 2+\text { tds.dflux } \\
\text { cpy`2+tds.dflux_cpz^2) }\end{array}$ & $\mathrm{mol} /\left(\mathrm{m}^{2} \cdot \mathrm{s}\right)$ & $\begin{array}{l}\text { Diffusive flux } \\
\text { magnitude }\end{array}$ & Domain 1 & \\
\hline $\begin{array}{l}\text { tds.tfluxMa } \\
\text { g_cp }\end{array}$ & $\begin{array}{l}\text { sqrt(tds.tflux_cpx }{ }^{\wedge} 2+\text { tds.tflux_ } \\
\text { cpy^2+tds.tflux_cpz`2) }\end{array}$ & $\mathrm{mol} /\left(\mathrm{m}^{2} \cdot \mathrm{s}\right)$ & $\begin{array}{l}\text { Total flux } \\
\text { magnitude }\end{array}$ & Domain 1 & \\
\hline $\begin{array}{l}\text { tds.tflux_cb } \\
\text { ufferx }\end{array}$ & $\begin{array}{l}\text { tds.dflux_cbufferx+tds.cflux_c } \\
\text { bufferx }\end{array}$ & $\mathrm{mol} /\left(\mathrm{m}^{2} \cdot \mathrm{s}\right)$ & $\begin{array}{l}\text { Total flux, } \mathrm{x} \\
\text { component }\end{array}$ & Domain 1 & $\begin{array}{l}+ \text { operati } \\
\text { on }\end{array}$ \\
\hline $\begin{array}{l}\text { tds.tflux_cb } \\
\text { uffery }\end{array}$ & $\begin{array}{l}\text { tds.dflux_cbuffery+tds.cflux_c } \\
\text { buffery }\end{array}$ & $\mathrm{mol} /\left(\mathrm{m}^{2} \cdot \mathrm{s}\right)$ & $\begin{array}{l}\text { Total flux, y } \\
\text { component }\end{array}$ & Domain 1 & $\begin{array}{l}+ \text { operati } \\
\text { on }\end{array}$ \\
\hline $\begin{array}{l}\text { tds.tflux_cb } \\
\text { ufferz }\end{array}$ & $\begin{array}{l}\text { tds.dflux_cbufferz+tds.cflux_c } \\
\text { bufferz }\end{array}$ & $\mathrm{mol} /\left(\mathrm{m}^{2} \cdot \mathrm{s}\right)$ & $\begin{array}{l}\text { Total flux, z } \\
\text { component }\end{array}$ & Domain 1 & $\begin{array}{l}+ \text { operati } \\
\text { on }\end{array}$ \\
\hline $\begin{array}{l}\text { tds.dfluxM } \\
\text { ag_cbuffer }\end{array}$ & $\begin{array}{l}\text { sqrt(tds.dflux_cbufferx }{ }^{\wedge} 2+\text { tds.d } \\
\text { flux_cbuffery^} 2+\text { tds.dflux_cbu } \\
\left.\text { fferz }^{\wedge} 2\right)\end{array}$ & $\mathrm{mol} /\left(\mathrm{m}^{2} \cdot \mathrm{s}\right)$ & $\begin{array}{l}\text { Diffusive flux } \\
\text { magnitude }\end{array}$ & Domain 1 & \\
\hline $\begin{array}{l}\text { tds.tfluxMa } \\
\text { g_cbuffer }\end{array}$ & $\begin{array}{l}\text { sqrt(tds.tflux_cbufferx }{ }^{\wedge} 2+\text { tds.tf } \\
\text { lux_cbuffery } 2+\text { tds.tflux_cbuff } \\
\left.\text { erz }^{\wedge} 2\right)\end{array}$ & $\mathrm{mol} /\left(\mathrm{m}^{2} \cdot \mathrm{s}\right)$ & $\begin{array}{l}\text { Total flux } \\
\text { magnitude }\end{array}$ & Domain 1 & \\
\hline
\end{tabular}




\begin{tabular}{|c|c|c|c|c|c|}
\hline Name & Expression & Unit & Description & Selection & Details \\
\hline $\begin{array}{l}\text { tds.dflux_c } \\
\text { px }\end{array}$ & $\begin{array}{l}\text {-tds.D_cpxx*cpx- } \\
\text { tds.D_cpxy*cpy }\end{array}$ & $\mathrm{mol} /\left(\mathrm{m}^{2} \cdot \mathrm{s}\right)$ & $\begin{array}{l}\text { Diffusive flux, } \mathrm{x} \\
\text { component }\end{array}$ & Domain 1 & \\
\hline $\begin{array}{l}\text { tds.dflux_c } \\
\text { py }\end{array}$ & $\begin{array}{l}\text {-tds.D_cpyx*cpx- } \\
\text { tds.D_cpyy*cpy }\end{array}$ & $\mathrm{mol} /\left(\mathrm{m}^{2} \cdot \mathrm{s}\right)$ & $\begin{array}{l}\text { Diffusive flux, y } \\
\text { component }\end{array}$ & Domain 1 & \\
\hline $\begin{array}{l}\text { tds.dflux_c } \\
\text { pz }\end{array}$ & $\begin{array}{l}\text {-tds.D_cpzx*cpx- } \\
\text { tds.D_cpzy*cpy }\end{array}$ & $\mathrm{mol} /\left(\mathrm{m}^{2} \cdot \mathrm{s}\right)$ & $\begin{array}{l}\text { Diffusive flux, z } \\
\text { component }\end{array}$ & Domain 1 & \\
\hline $\begin{array}{l}\text { tds.grad_cp } \\
x\end{array}$ & $\operatorname{cpx}$ & $\mathrm{mol} / \mathrm{m}^{4}$ & $\begin{array}{l}\text { Concentration } \\
\text { gradient, } \mathrm{x} \\
\text { component }\end{array}$ & Domain 1 & \\
\hline $\begin{array}{l}\text { tds.grad_cp } \\
\text { y }\end{array}$ & cpy & $\mathrm{mol} / \mathrm{m}^{4}$ & $\begin{array}{l}\text { Concentration } \\
\text { gradient, y } \\
\text { component }\end{array}$ & Domain 1 & \\
\hline $\begin{array}{l}\text { tds.grad_cp } \\
z\end{array}$ & 0 & $\mathrm{~mol} / \mathrm{m}^{4}$ & $\begin{array}{l}\text { Concentration } \\
\text { gradient, } \mathrm{z} \\
\text { component }\end{array}$ & Domain 1 & \\
\hline $\begin{array}{l}\text { tds.dflux_c } \\
\text { bufferx }\end{array}$ & $\begin{array}{l}\text {-tds.D_cbufferxx*cbufferx- } \\
\text { tds.D_cbufferxy*cbuffery }\end{array}$ & $\mathrm{mol} /\left(\mathrm{m}^{2} \cdot \mathrm{s}\right)$ & $\begin{array}{l}\text { Diffusive flux, } \mathrm{x} \\
\text { component }\end{array}$ & Domain 1 & \\
\hline $\begin{array}{l}\text { tds.dflux_c } \\
\text { buffery }\end{array}$ & $\begin{array}{l}\text {-tds.D_cbufferyx*cbufferx- } \\
\text { tds.D_cbufferyy*cbuffery }\end{array}$ & $\mathrm{mol} /\left(\mathrm{m}^{2} \cdot \mathrm{s}\right)$ & $\begin{array}{l}\text { Diffusive flux, y } \\
\text { component }\end{array}$ & Domain 1 & \\
\hline $\begin{array}{l}\text { tds.dflux_c } \\
\text { bufferz }\end{array}$ & $\begin{array}{l}\text {-tds.D_cbufferzx*cbufferx- } \\
\text { tds.D_cbufferzy*cbuffery }\end{array}$ & $\mathrm{mol} /\left(\mathrm{m}^{2} \cdot \mathrm{s}\right)$ & $\begin{array}{l}\text { Diffusive flux, z } \\
\text { component }\end{array}$ & Domain 1 & \\
\hline $\begin{array}{l}\text { tds.grad_cb } \\
\text { ufferx }\end{array}$ & cbufferx & $\mathrm{mol} / \mathrm{m}^{4}$ & $\begin{array}{l}\text { Concentration } \\
\text { gradient, } \mathrm{x} \\
\text { component }\end{array}$ & Domain 1 & \\
\hline $\begin{array}{l}\text { tds.grad_cb } \\
\text { uffery }\end{array}$ & cbuffery & $\mathrm{mol} / \mathrm{m}^{4}$ & $\begin{array}{l}\text { Concentration } \\
\text { gradient, y } \\
\text { component }\end{array}$ & Domain 1 & \\
\hline $\begin{array}{l}\text { tds.grad_cb } \\
\text { ufferz }\end{array}$ & 0 & $\mathrm{~mol} / \mathrm{m}^{4}$ & $\begin{array}{l}\text { Concentration } \\
\text { gradient, } \mathrm{z} \\
\text { component }\end{array}$ & Domain 1 & \\
\hline $\begin{array}{l}\text { tds.cflux_c } \\
\text { px }\end{array}$ & cp*tds.u & $\mathrm{mol} /\left(\mathrm{m}^{2} \cdot \mathrm{s}\right)$ & $\begin{array}{l}\text { Convective flux, } \\
\text { x component }\end{array}$ & Domain 1 & \\
\hline $\begin{array}{l}\text { tds.cflux_c } \\
\text { py }\end{array}$ & cp*tds.v & $\mathrm{mol} /\left(\mathrm{m}^{2} \cdot \mathrm{s}\right)$ & $\begin{array}{l}\text { Convective flux, } \\
\text { y component }\end{array}$ & Domain 1 & \\
\hline $\begin{array}{l}\text { tds.cflux_c } \\
\text { pz }\end{array}$ & cp*tds.w & $\mathrm{mol} /\left(\mathrm{m}^{2} \cdot \mathrm{s}\right)$ & $\begin{array}{l}\text { Convective flux, } \\
\text { z component }\end{array}$ & Domain 1 & \\
\hline $\begin{array}{l}\text { tds.cfluxMa } \\
\text { g_cp }\end{array}$ & $\begin{array}{l}\text { sqrt(tds.cflux_cpx }{ }^{\wedge} 2+\text { tds.cflux } \\
\text { cpy^} 2+\text { tds.cflux_cpz } \\
\text { 2 } 2)\end{array}$ & $\mathrm{mol} /\left(\mathrm{m}^{2} \cdot \mathrm{s}\right)$ & $\begin{array}{l}\text { Convective flux } \\
\text { magnitude }\end{array}$ & Domain 1 & \\
\hline $\begin{array}{l}\text { tds.cflux_c } \\
\text { bufferx }\end{array}$ & cbuffer*tds.u & $\mathrm{mol} /\left(\mathrm{m}^{2} \cdot \mathrm{s}\right)$ & $\begin{array}{l}\text { Convective flux, } \\
\text { x component }\end{array}$ & Domain 1 & \\
\hline $\begin{array}{l}\text { tds.cflux_c } \\
\text { buffery }\end{array}$ & cbuffer*tds.v & $\mathrm{mol} /\left(\mathrm{m}^{2} \cdot \mathrm{s}\right)$ & $\begin{array}{l}\text { Convective flux, } \\
\text { y component }\end{array}$ & Domain 1 & \\
\hline $\begin{array}{l}\text { tds.cflux_c } \\
\text { bufferz }\end{array}$ & cbuffer*tds.w & $\mathrm{mol} /\left(\mathrm{m}^{2} \cdot \mathrm{s}\right)$ & $\begin{array}{l}\text { Convective flux, } \\
\text { z component }\end{array}$ & Domain 1 & \\
\hline $\begin{array}{l}\text { tds.cfluxMa } \\
\text { g_cbuffer }\end{array}$ & $\begin{array}{l}\text { sqrt(tds.cflux_cbufferx }{ }^{\wedge} 2+\text { tds.c } \\
\text { flux_cbuffery^} 2+\text { tds.cflux_cbuf } \\
\left.\text { ferz }^{\wedge} 2\right)\end{array}$ & $\mathrm{mol} /\left(\mathrm{m}^{2} \cdot \mathrm{s}\right)$ & $\begin{array}{l}\text { Convective flux } \\
\text { magnitude }\end{array}$ & Domain 1 & \\
\hline $\begin{array}{l}\text { tds.bndFlux } \\
\text { cp }\end{array}$ & -dflux_spatial(cp) & $\mathrm{mol} /\left(\mathrm{m}^{2} \cdot \mathrm{s}\right)$ & Boundary flux & $\begin{array}{l}\text { Boundaries } \\
1-12\end{array}$ & \\
\hline $\begin{array}{l}\text { tds.bndFlux } \\
\text { cbuffer }\end{array}$ & -dflux_spatial(cbuffer) & $\mathrm{mol} /\left(\mathrm{m}^{2} \cdot \mathrm{s}\right)$ & Boundary flux & $\begin{array}{l}\text { Boundaries } \\
1-12\end{array}$ & \\
\hline
\end{tabular}




\begin{tabular}{|l|l|l|l|l|l|}
\hline Name & Expression & Unit & Description & Selection & Details \\
\hline tds.Res_cp & $\begin{array}{l}\mathrm{d}(\mathrm{cp}, \mathrm{t})+\mathrm{tds} . \mathrm{u}^{*} \mathrm{cpx}+\mathrm{tds} . \mathrm{v}^{*} \mathrm{cpy}- \\
\mathrm{tds} \cdot \mathrm{R} \_\mathrm{cp}\end{array}$ & $\mathrm{mol} /\left(\mathrm{m}^{3} \cdot \mathrm{s}\right)$ & $\begin{array}{l}\text { Equation } \\
\text { residual }\end{array}$ & Domain 1 \\
\hline $\begin{array}{l}\text { tds.Res_cb } \\
\text { uffer }\end{array}$ & $\begin{array}{l}\mathrm{d}(\mathrm{cbuffer}, \mathrm{t})+\mathrm{tds} . \mathrm{u}^{*} \text { cbufferx }+\mathrm{tds} \\
. \mathrm{v}^{*} \text { cbuffery-tds.R_cbuffer }\end{array}$ & $\mathrm{mol} /\left(\mathrm{m}^{3} \cdot \mathrm{s}\right)$ & $\begin{array}{l}\text { Equation } \\
\text { residual }\end{array}$ & Domain 1 & \\
\hline
\end{tabular}

Shape functions

\begin{tabular}{|l|l|l|l|l|l|}
\hline Name & Shape function & Unit & Description & Shape frame & Selection \\
\hline $\mathrm{cp}$ & Lagrange (Linear) & $\mathrm{mol} / \mathrm{m}^{3}$ & Concentration & Spatial & Domain 1 \\
\hline cbuffer & Lagrange (Linear) & $\mathrm{mol} / \mathrm{m}^{3}$ & Concentration & Spatial & Domain 1 \\
\hline
\end{tabular}

Weak expressions

\begin{tabular}{|c|c|c|c|}
\hline Weak expression & $\begin{array}{l}\text { Integration } \\
\text { order }\end{array}$ & $\begin{array}{l}\text { Integration } \\
\text { frame }\end{array}$ & Selection \\
\hline $\begin{array}{l}\text { - } \\
\text { py) } \\
\text { py }\end{array}$ & 2 & Spatial & Domain 1 \\
\hline $\begin{array}{l}- \\
\text { cbuffert*test(cbuffer)+tds.dflux_cbufferx*test(cbufferx)+t } \\
\text { ds.dflux_cbuffery*test(cbuffery) }\end{array}$ & 2 & Spatial & Domain 1 \\
\hline $\begin{array}{l}\left.\text { (tds.u*cpx+tds. } v^{*} \text { cpy }\right)^{*} \text { test }(\mathrm{cp}) *(\text { isScalingSystemDomain }= \\
=0)\end{array}$ & 2 & Spatial & Domain 1 \\
\hline tds.cbf_cp*test(cp) & 2 & Spatial & $\begin{array}{l}\text { Boundaries } \\
1-12\end{array}$ \\
\hline $\begin{array}{l}- \\
\left.\left(\text { tds.u*cbufferx }+ \text { tds. } v^{*} \text { cbuffery }\right) * \text { test(cbuffer }\right) *(\text { is ScalingS } \\
\text { ystemDomain }==0)\end{array}$ & 2 & Spatial & Domain 1 \\
\hline tds.cbf_cbuffer*test(cbuffer) & 2 & Spatial & $\begin{array}{l}\text { Boundaries } \\
1-12\end{array}$ \\
\hline tds.streamline $*($ isScalingSystemDomain $==0)$ & 2 & Spatial & Domain 1 \\
\hline tds.crosswind $*$ (isScalingSystemDomain $==0$ ) & 4 & Spatial & Domain 1 \\
\hline
\end{tabular}

No Flux 1

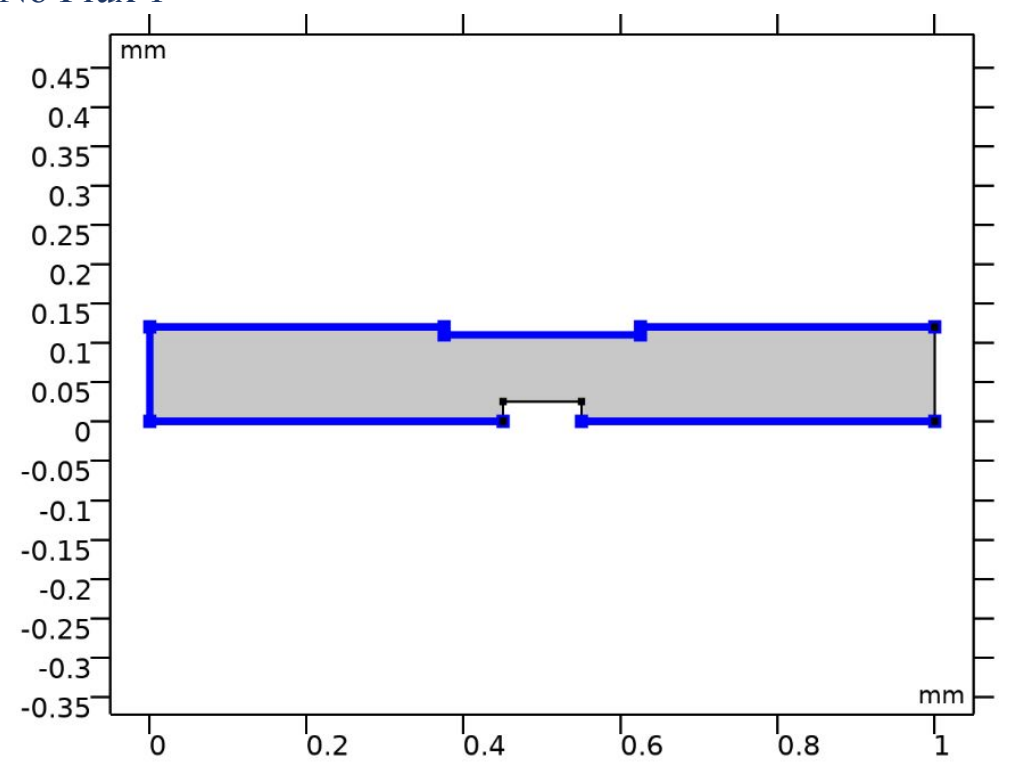

No Flux 1 
Selection

Geometric entity level Boundary

Selection Geometry geom1: Dimension 1: All boundaries

Equations

$-\mathbf{n} \cdot\left(\mathbf{J}_{i}+\mathbf{u} c_{i}\right)=0$

Convection

Settings

\section{Description Value}

Include On

Variables

\begin{tabular}{|l|l|l|l|l|}
\hline Name & Expression & Unit & Description & Selection \\
\hline tds.cbf_cp & $\begin{array}{l}\text { cp*(tds.u*tds.nxmesh+tds. } v^{*} \text { tds.nymesh }+\mathrm{t} \\
\text { ds.w*tds.nzmesh })\end{array}$ & $\mathrm{mol} /\left(\mathrm{m}^{2} \cdot \mathrm{s}\right)$ & $\begin{array}{l}\text { Convective } \\
\text { boundary flux }\end{array}$ & $\begin{array}{l}\text { Boundaries } \\
1-5,9-11\end{array}$ \\
\hline $\begin{array}{l}\text { tds.cbf_cbuf } \\
\text { fer }\end{array}$ & $\begin{array}{l}\text { cbuffer*(tds.u*tds.nxmesh+tds. }{ }^{*} \text { tds.nyme } \\
\text { sh+tds.w*tds.nzmesh })\end{array}$ & $\mathrm{mol} /\left(\mathrm{m}^{2} \cdot \mathrm{s}\right)$ & $\begin{array}{l}\text { Convective } \\
\text { boundary flux }\end{array}$ & $\begin{array}{l}\text { Boundaries } \\
1-5,9-11\end{array}$ \\
\hline
\end{tabular}

Initial Values 1

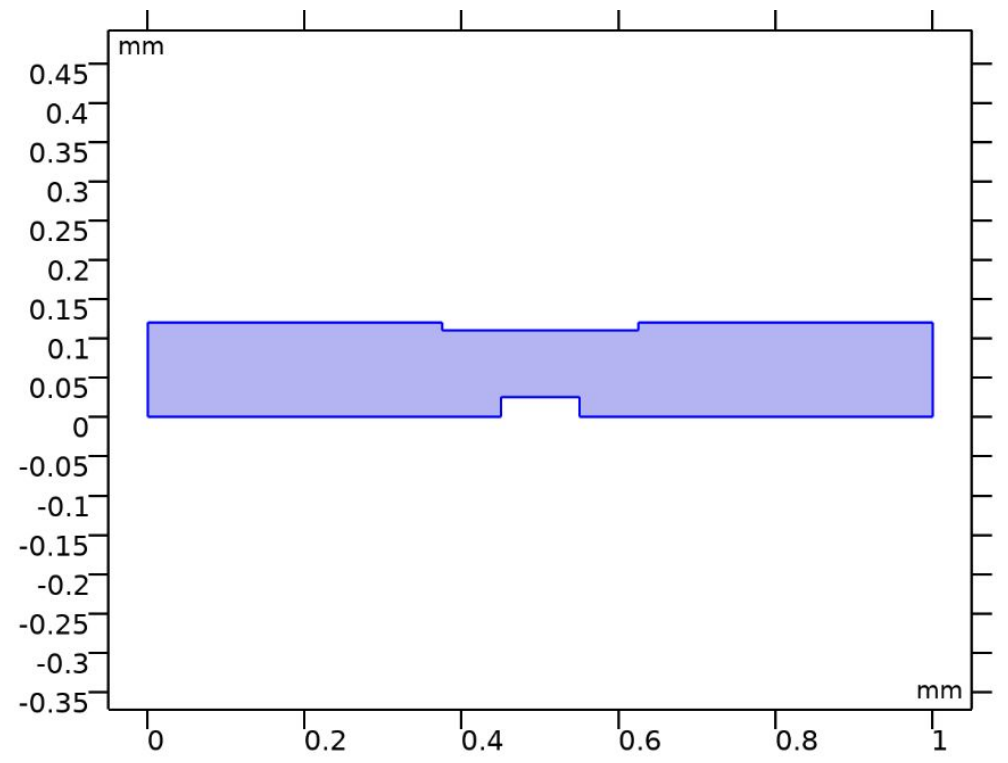

Initial Values 1

Selection

\begin{tabular}{|l|l|}
\hline Geometric entity level & Domain \\
\hline Selection & Geometry geom1: Dimension 2: All domains \\
\hline
\end{tabular}

Initial values

Settings

\begin{tabular}{l|l} 
Description & Value \\
\hline Concentration & $\{$ c_proton 0, c_buffer \\
\hline
\end{tabular}


Inflow 1

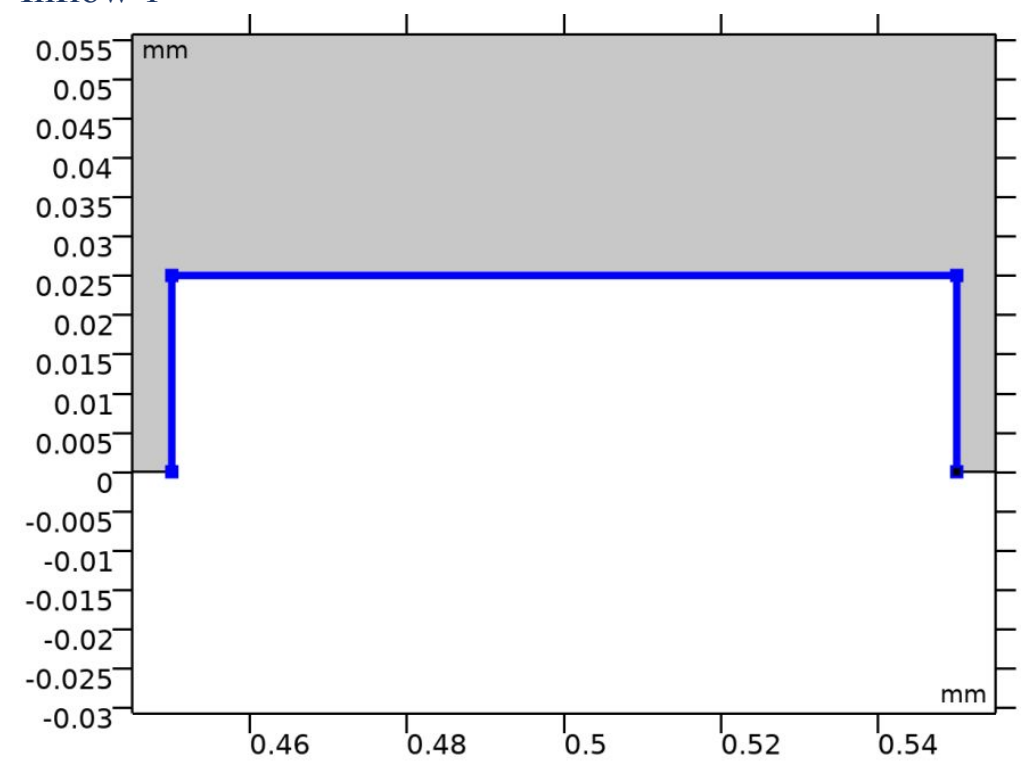

Inflow 1

Selection

\begin{tabular}{|l|l|}
\hline Geometric entity level & Boundary \\
\hline Selection & Geometry geom1: Dimension 1: Boundaries 6-8 \\
\hline
\end{tabular}

Equations

$c_{i}=c_{0, j}$

Concentration

Settings

\begin{tabular}{|l|l|}
\hline Description & Value \\
\hline Concentration & $\{$ c_proton, 0$\}$ \\
\hline
\end{tabular}

Boundary condition type

Settings

\begin{tabular}{|l|l|}
\hline Description & Value \\
\hline Boundary condition type & Concentration constraint \\
\hline
\end{tabular}

Variables

\begin{tabular}{|c|c|c|c|c|c|}
\hline Name & Expression & Unit & Description & Selection & Details \\
\hline tds.c0_cp & c_proton & $\mathrm{mol} / \mathrm{m}^{3}$ & Concentration & $\begin{array}{l}\text { Boundaries } \\
6-8\end{array}$ & $\begin{array}{l}+ \text { operati } \\
\text { on }\end{array}$ \\
\hline $\begin{array}{l}\text { tds.c0_cbuf } \\
\text { fer }\end{array}$ & 0 & $\mathrm{~mol} / \mathrm{m}^{3}$ & Concentration & $\begin{array}{l}\text { Boundaries } \\
6-8\end{array}$ & $\begin{array}{l}+ \text { operati } \\
\text { on }\end{array}$ \\
\hline $\begin{array}{l}\operatorname{tds} . i n 1 . c 0 \_a \\
\operatorname{vg\_ cp}\end{array}$ & 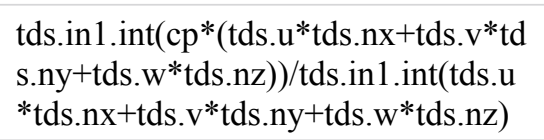 & $\mathrm{mol} / \mathrm{m}^{3}$ & Concentration & Global & \\
\hline $\begin{array}{l}\text { tds.in1.c0_a } \\
\text { vg_cbuffer }\end{array}$ & 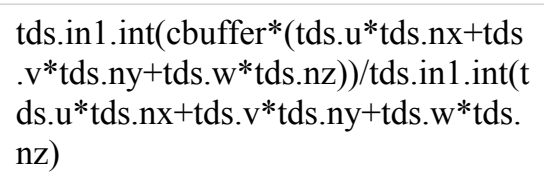 & $\mathrm{mol} / \mathrm{m}^{3}$ & Concentration & Global & \\
\hline
\end{tabular}


Constraints

\begin{tabular}{|l|l|l|l|l|}
\hline Constraint & Constraint force & Shape function & Selection & Details \\
\hline -cp+tds.c0_cp & -test(cp) & Lagrange (Linear) & Boundaries 6-8 & Elemental \\
\hline $\begin{array}{l}\text { cbuffer+tds.c0_cbuffer } \\
\text { cbest(cbuffer) }\end{array}$ & Lagrange (Linear) & Boundaries 6-8 & Elemental \\
\hline
\end{tabular}

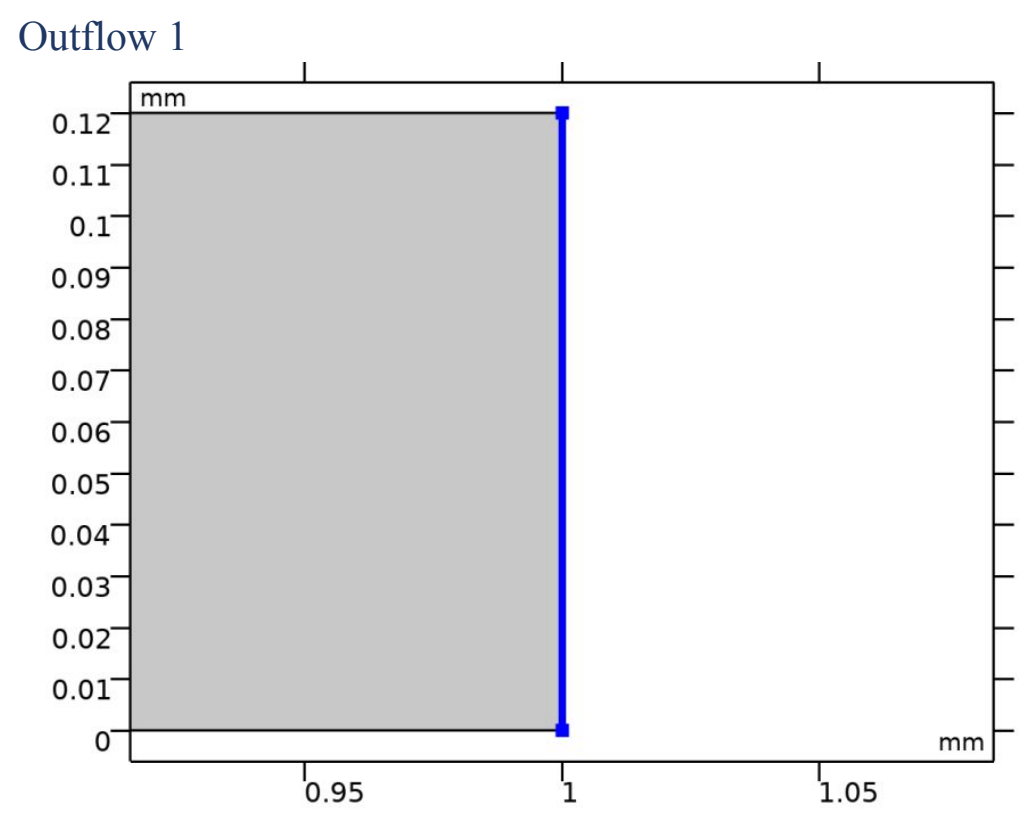

Outflow 1

\section{Selection}

Geometric entity level Boundary

Selection

Geometry geom1: Dimension 1: Boundary 12

\section{Equations}

$$
\mathbf{n} \cdot D_{i} \nabla c_{i}=0
$$

\section{Variables}

\begin{tabular}{|c|c|c|c|c|}
\hline Name & Expression & Unit & Description & Selection \\
\hline $\begin{array}{l}\text { tds.out1.c0_- } \\
\text { avg_cp }\end{array}$ & 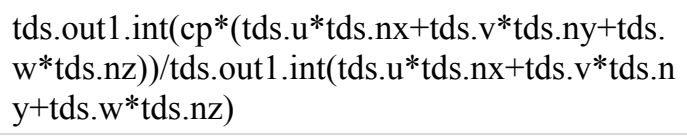 & $\mathrm{mol} / \mathrm{m}^{3}$ & Concentration & Global \\
\hline $\begin{array}{l}\text { tds.out } 1 . c 0 \_ \\
\text {avg_cbuffer }\end{array}$ & $\begin{array}{l}\text { tds.out1.int(cbuffer*(tds.u*tds.nx+tds.v*tds.ny } \\
+ \text { tds.w*tds.nz) }) / \text { tds.out1.int(tds.u*tds.nx+tds.v } \\
\text { *tds.ny+tds.w*tds.nz) }\end{array}$ & $\mathrm{mol} / \mathrm{m}^{3}$ & Concentration & Global \\
\hline
\end{tabular}

Weak expressions

\begin{tabular}{|l|l|l|l|}
\hline Weak expression & Integration order & Integration frame & Selection \\
\hline 0 & 2 & Spatial & Boundary 12 \\
\hline 0 & 2 & Spatial & Boundary 12 \\
\hline
\end{tabular}




\section{Mesh 1}

Mesh statistics

\begin{tabular}{|l|l|}
\hline Description & Value \\
\hline Minimum element quality & 0.8946 \\
\hline Average element quality & 0.9894 \\
\hline Triangle & 2995 \\
\hline Edge element & 233 \\
\hline Vertex element & 12 \\
\hline
\end{tabular}

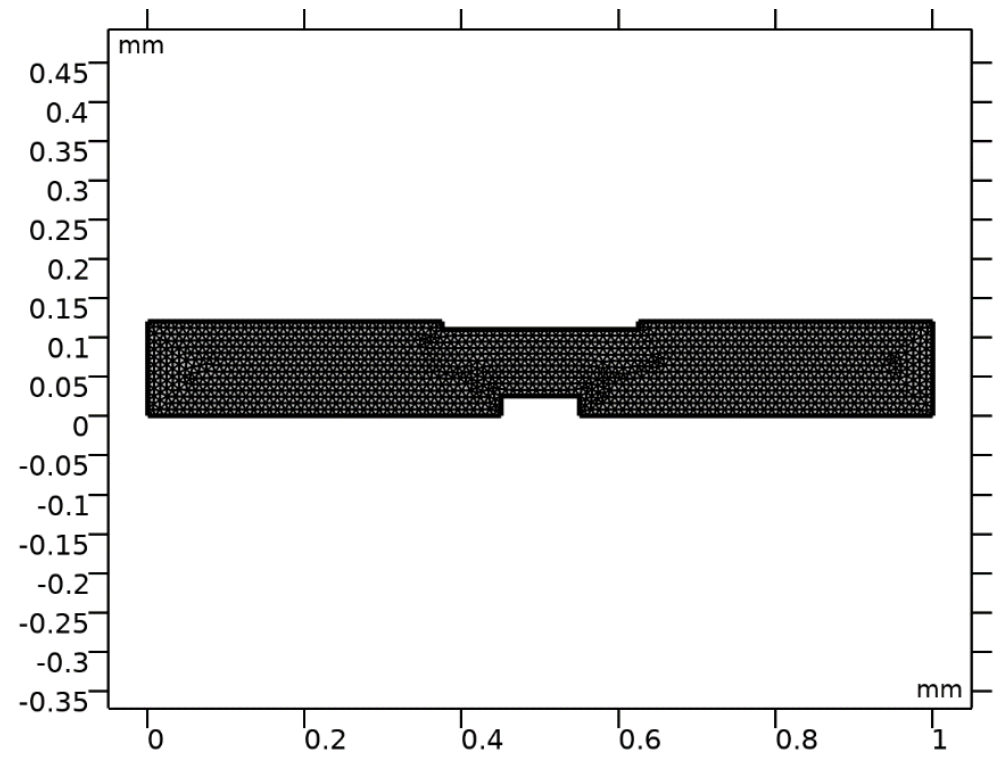

Mesh 1

Size (size)

Settings

\begin{tabular}{|l|l|}
\hline Description & Value \\
\hline Maximum element size & 0.01 \\
\hline Minimum element size & $2.0 \mathrm{E}-5$ \\
\hline Curvature factor & 0.2 \\
\hline Predefined size & Extremely fine \\
\hline
\end{tabular}

Free Triangular 1 (ftri1)

Selection

Geometric entity level Remaining 


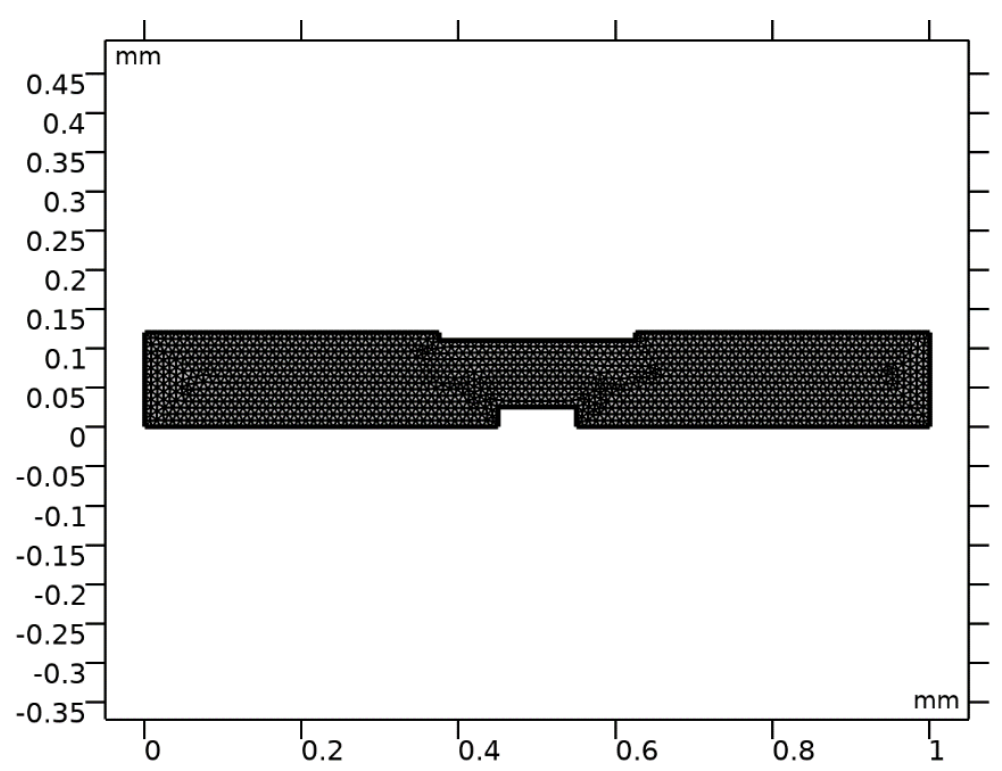

Free Triangular 1

\section{Study}

Computation information

\begin{tabular}{|l|l|}
\hline Computation time & $6 \mathrm{~s}$ \\
\hline CPU & Intel64 Family 6 Model 142 Stepping 12, 4 cores \\
\hline Operating system & Windows 10 \\
\hline
\end{tabular}

\section{Time Dependent}

\begin{tabular}{|l|l|}
\hline Times & Unit \\
\hline range $(0,0.5,30)$ & $\mathrm{s}$ \\
\hline
\end{tabular}

Study settings

\begin{tabular}{|l|l|}
\hline Description & Value \\
\hline Include geometric nonlinearity & Off \\
\hline
\end{tabular}

\begin{tabular}{|l|l|}
\hline Description & Value \\
\hline \multirow{3}{*}{ Times } & $\{0,0.5,1,1.5,2,2.5,3,3.5,4,4.5,5,5.5,6,6.5,7,7.5,8,8.5,9,9.5,10,10.5,11,11.5,12$ \\
& $12.5,13,13.5,14,14.5,15,15.5,16,16.5,17,17.5,18,18.5,19,19.5,20,20.5,21,21.5$ \\
& $22,22.5,23,23.5,24,24.5,25,25.5,26,26.5,27,27.5,28,28.5,29,29.5,30\}$ \\
\hline
\end{tabular}

Physics and variables selection

\section{Physics interface Discretization \\ Transport of Diluted Species (tds) physics}

Mesh selection

\begin{tabular}{|l|l|}
\hline Geometry & Mesh \\
\hline Geometry 1 (geom1) & mesh1 \\
\hline
\end{tabular}




\section{Solution}

Compile Equations: Time Dependent (st1)

Study and step

\begin{tabular}{|l|l|}
\hline Description & Value \\
\hline Use study & $\underline{\text { Study }}$ \\
\hline Use study step & $\underline{\text { Time Dependent }}$ \\
\hline
\end{tabular}

Dependent Variables 1 (v1)

General

\begin{tabular}{|l|l|}
\hline Description & Value \\
\hline Defined by study step & $\underline{\text { Time Dependent }}$ \\
\hline
\end{tabular}

Residual scaling

\begin{tabular}{|l|l|}
\hline Description & Value \\
\hline Method & Manual
\end{tabular}

Initial value calculation constants

\begin{tabular}{|l|l|}
\hline Constant name & Initial value source \\
\hline $\mathrm{t}$ & range $(0,0.5,30)$ \\
\hline timestep & $0.03[\mathrm{~s}]$ \\
\hline
\end{tabular}

Concentration (comp1.cbuffer) (comp1_cbuffer)

General

\begin{tabular}{|l|l|} 
Description & Value \\
\hline Field components & comp1.cbuffer \\
\hline Internal variables & $\{$ comp1.uflux.cbuffer, comp1.dflux.cbuffer, comp1.tds.dt2Inv_cbuffer \\
\hline
\end{tabular}

Concentration (comp1.cp) (comp1_cp)

General

\begin{tabular}{|l|l|}
\hline Description & Value \\
\hline Field components & comp1.cp \\
\hline Internal variables & $\{$ comp1.uflux.cp, comp1.dflux.cp, comp1.tds.dt2Inv_cp \\
\hline
\end{tabular}

Time-Dependent Solver 1 (t1)

General

\begin{tabular}{|l|l|}
\hline Description & Value \\
\hline Defined by study step & $\underline{\text { Time Dependent }}$ \\
\hline \multirow{2}{*}{ Times } & $\{0,0.5,1,1.5,2,2.5,3,3.5,4,4.5,5,5.5,6,6.5,7,7.5,8,8.5,9,9.5,10$, \\
& $10.5,11,11.5,12,12.5,13,13.5,14,14.5,15,15.5,16,16.5,17,17.5,18$, \\
& $18.5,19,19.5,20,20.5,21,21.5,22,22.5,23,23.5,24,24.5,25,25.5,26$, \\
\hline Relative tolerance & $26.5,27,27.5,28,28.5,29,29.5,30\}$ \\
\hline & 0.005 \\
\hline
\end{tabular}

Time stepping

\begin{tabular}{|l|l|}
\hline Description & Value \\
\hline Maximum BDF order & 2 \\
\hline
\end{tabular}


Fully Coupled 1 (fc1)

General

\begin{tabular}{|l|l|} 
Description & Value \\
\hline Linear solver & $\underline{\text { Direct 1 }}$ \\
\hline
\end{tabular}

Method and termination

\begin{tabular}{|l|l|}
\hline Description & Value \\
\hline Damping factor & 0.9 \\
\hline Jacobian update & Once per time step \\
\hline Maximum number of iterations & 8 \\
\hline Stabilization and acceleration & Anderson acceleration \\
\hline Dimension of iteration space & 5 \\
\hline Mixing parameter & 0.9 \\
\hline Iteration delay & 1 \\
\hline
\end{tabular}

Direct 1 (d1)

General

\begin{tabular}{|l|l|}
\hline Description & Value \\
\hline Solver & PARDISO \\
\hline Pivoting perturbation & $1.0 \mathrm{E}-13$ \\
\hline
\end{tabular}

\section{Results}

Datasets

Study /Solution

\section{Solution}

\begin{tabular}{|l|l|} 
Description & Value \\
\hline Solution & $\underline{\text { Solution }}$ \\
\hline Component & Save Point Geometry 1 \\
\hline
\end{tabular}

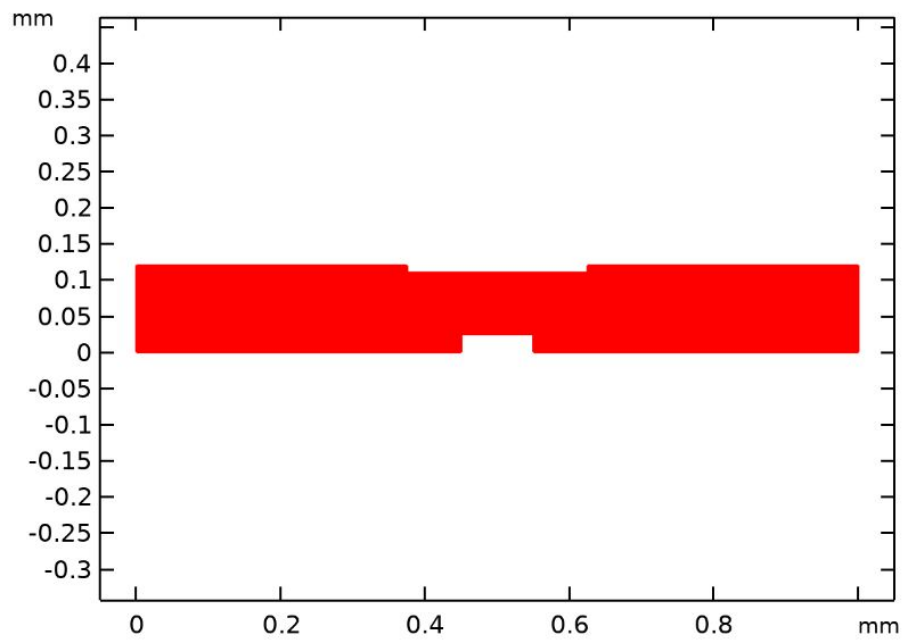

Dataset: Study/Solution 
Cut Line 2D 1

Data

\begin{tabular}{|l|l|}
\hline Description & Value \\
\hline Dataset & Study /Solution \\
\hline
\end{tabular}

Line data

\begin{tabular}{l|l} 
Description & Value \\
\hline Line entry method & Two points \\
\hline Points & $\{\{0.500001,0\},\{0.500001,0.12000000000000002\}\}$
\end{tabular}

Advanced

\begin{tabular}{|l|l|} 
Description & Value \\
\hline Space variable & $\operatorname{cln} 1 \mathrm{x}$ \\
\hline Normal variables & $\{\mathrm{cln} 1 \mathrm{nx}, \operatorname{cln} 1 \mathrm{n} y\}$ \\
\hline
\end{tabular}

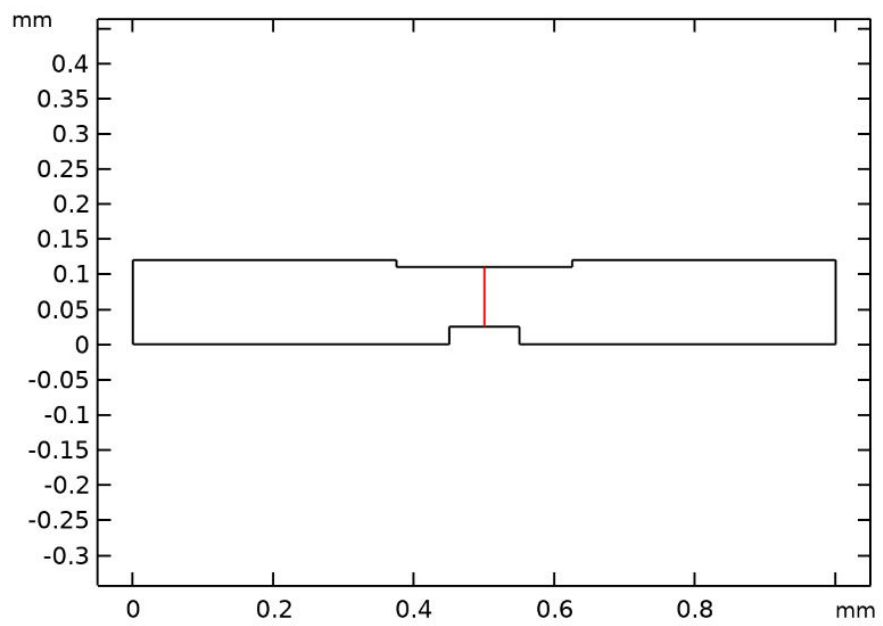

Dataset: Cut Line 2D 1

Tables

Evaluation 2D

Interactive $2 \mathrm{D}$ values

\begin{tabular}{|l|l|l|}
\hline $\mathbf{x}$ & $\mathbf{y}$ & Value \\
\hline 0.43245 & 0.10225 & $-1.3529 \mathrm{E}-7$ \\
\hline 0.48881 & 0.089264 & 6.7262 \\
\hline 0.16862 & 0.058279 & 7.1083 \\
\hline 0.025743 & 0.054836 & 7.1285 \\
\hline 0.90712 & 0.077214 & 7.1249 \\
\hline
\end{tabular}




\section{Plot Groups}

Diffusion over time

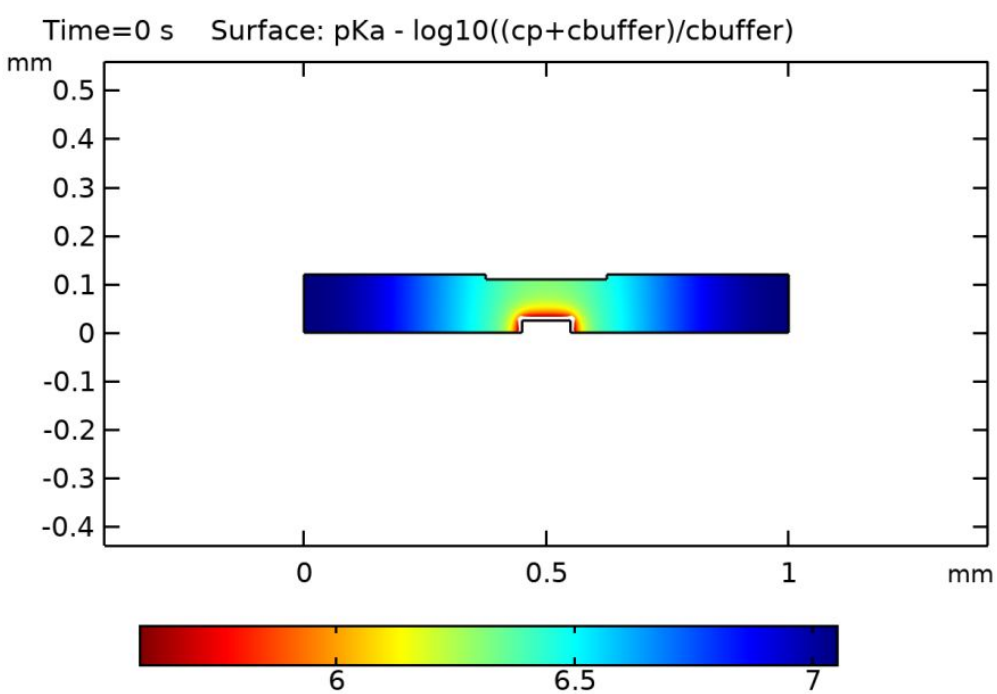

Surface: $p K a-\log 10((c p+c b u f f e r) / c b u f f e r)$

Sensorspot - Integral

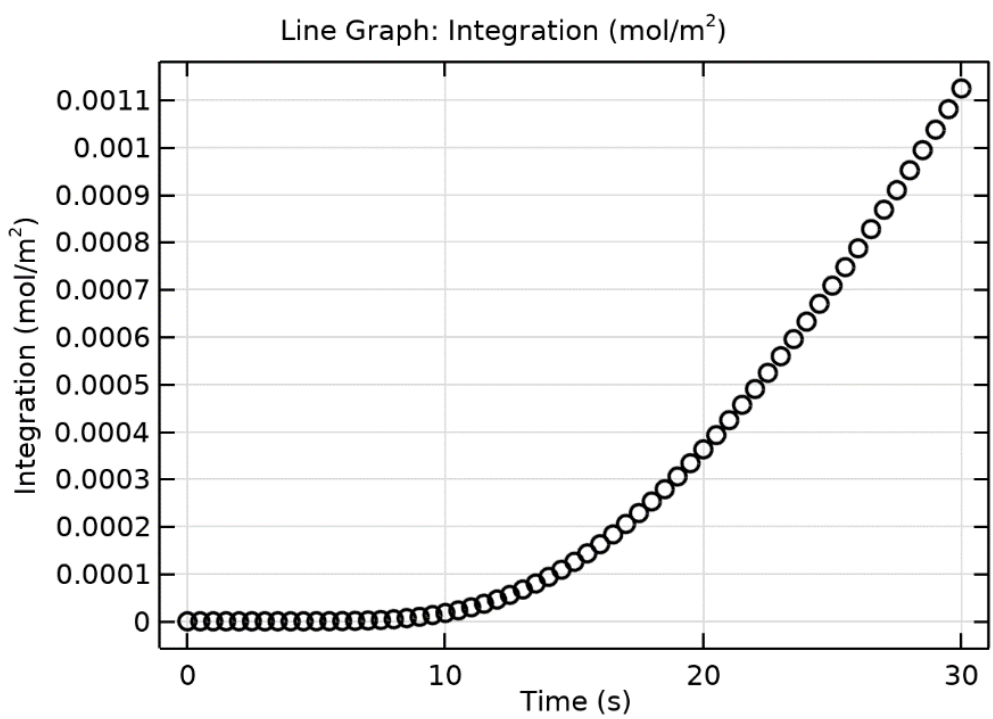

Line Graph: Integration $\left(\mathrm{mol} / \mathrm{m}^{2}\right)$ 
$\mathrm{pH}$ on sensorspot

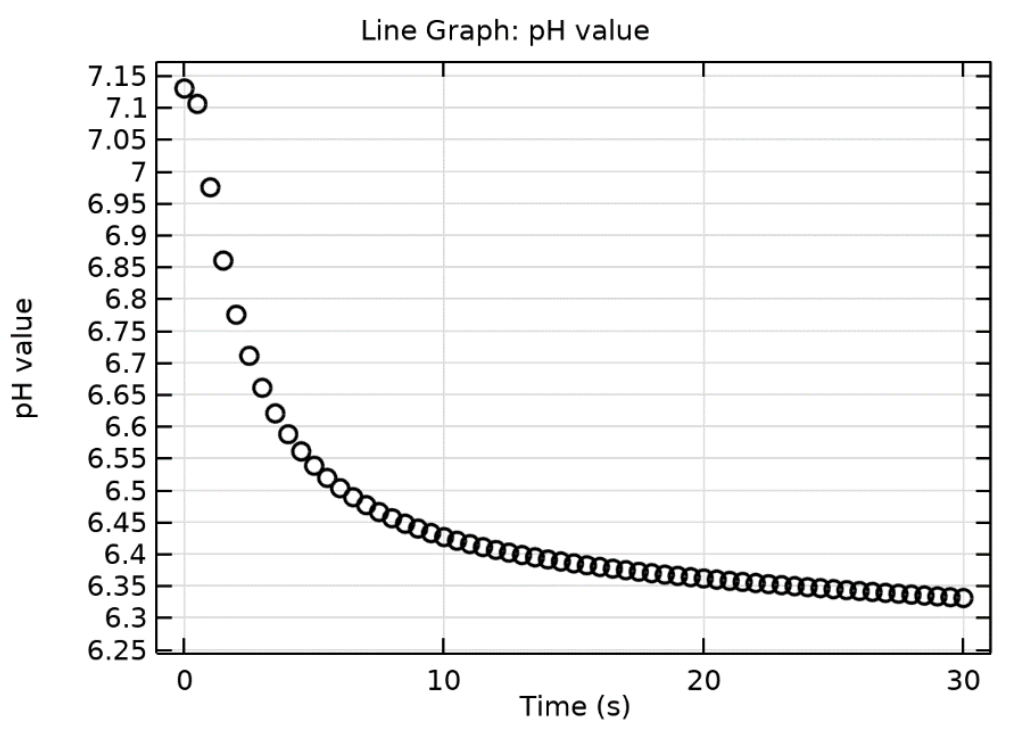

Line Graph: pH value 